\title{
Systems of small-noise stochastic reaction-diffusion equations satisfy a large deviations principle that is uniform over all initial data
}

\author{
M. Salins \\ Boston University \\ msalins@bu.edu
}

August 11, 2021

\begin{abstract}
Large deviations principles characterize the exponential decay rates of the probabilities of rare events. Cerrai and Röckner [13] proved that systems of stochastic reaction-diffusion equations satisfy a large deviations principle that is uniform over bounded sets of initial data.

This paper proves uniform large deviations results for a system of stochastic reaction-diffusion equations in a more general setting than Cerrai and Röckner. Furthermore, this paper identifies two common situations where the large deviations principle is uniform over unbounded sets of initial data, enabling the characterization of FreidlinWentzell exit time and exit shape asymptotics from unbounded sets.
\end{abstract}

\section{Introduction}

This paper investigates uniform large deviations principles for systems of stochastic reaction-diffusion equations. Let $\mathcal{O} \subset \mathbb{R}^{d}$ be a bounded open set with smooth boundary. Let $r \in \mathbb{N}$ be fixed. For $\varepsilon>0$, and continuous initial data $x: \mathcal{O} \times\{1, \ldots, r\} \rightarrow \mathbb{R}, X_{x}^{\varepsilon}(t, \xi)=\left(X_{x, 1}^{\varepsilon}(t, \xi), \ldots, X_{x, r}^{\varepsilon}(t, \xi)\right)$ is the $\mathbb{R}^{r}$-valued random field solution to the equations for $i \in\{1, \ldots, r\}$, 


$$
\left\{\begin{array}{l}
\frac{\partial X_{x, i}^{\varepsilon}(t, \xi)=\mathcal{A}_{i} X_{x, i}^{\varepsilon}(t, \xi)+f_{i}\left(t, \xi, X_{x}^{\varepsilon}(t, \xi)\right)}{r t} \\
\quad+\sqrt{\varepsilon} \sum_{n=1}^{r} \sigma_{i n}\left(t, \xi, X_{x}^{\varepsilon}(t, \xi)\right) \frac{\partial w_{n}}{\partial t}(t, \xi), \\
X_{x}^{\varepsilon}(t, \xi)=0, \quad \xi \in \partial \mathcal{O}, \quad t \geq 0 \\
X_{x}^{\varepsilon}(0, \xi)=x(\xi), \quad \xi \in \mathcal{O} .
\end{array}\right.
$$

In the above equation, $\left\{\mathcal{A}_{i}\right\}_{i=1}^{r}$ are elliptic second-order differential operators, $\sigma_{i n}$ are globally Lipschitz continuous in the third variable, and $f_{i}$ can be written as $f_{i}(t, \xi, x)=g_{i}\left(t, \xi, x_{i}\right)+h_{i}(t, \xi, x)$ where $g_{i}$ is continuous and non-increasing in its third argument and $h_{i}$ is globally Lipschitz continuous in its third argument. The multiplicative noise coefficients $\sigma_{\text {in }}$ are Lipschitz continuous in their third variable. The Gaussian noises $\frac{\partial w_{n}}{\partial t}$, defined on some probability space $(\Omega, \mathcal{F}, \mathbb{P})$ are white in time, but possibly correlated in space.

As $\varepsilon \rightarrow 0$, the stochastic perturbations disappear and the solutions converges to the solution of the unperturbed system of partial differential equations $X_{x}^{0}$. This convergence is uniform over finite time intervals in the sense that for any $T>0$,

$$
\lim _{\varepsilon \rightarrow 0} \sup _{t \in[0, T]} \sup _{\xi \in \mathcal{O}} \sup _{i \in\{1, \ldots, r\}}\left|X_{x, i}^{\varepsilon}(t, \xi)-X_{x, i}^{0}(t, \xi)\right|=0 \text { in probability. }
$$

Over unbounded time intervals, however, the stochastic system $X_{x}^{\varepsilon}$ will behave fundamentally differently than $X_{x}^{0}$ for any positive $\varepsilon>0$ if the $\sigma_{i n}$ terms are sufficiently non-degenerate. For example, let $D \subset C(\mathcal{O} \times\{1, \ldots, r\})$ be a collection of continuous functions that are invariant under the unperturbed dynamics. This means that if $x \in D$, then $X_{x}^{0}(t, \cdot) \in D$ for all $t>0$. Under reasonable assumptions on $D$ and $\sigma_{i n}$, one can show that for any $\varepsilon>0$, $X_{x}^{\varepsilon}$ exits $D$ with probability one. The Freidlin-Wentzell exit time problem characterizes the exponential divergence rate of the exit time

$$
\tau_{x}^{\varepsilon}:=\inf \left\{t>0: X_{x}^{\varepsilon}(t, \cdot) \notin D\right\}
$$

as well as the limiting behavior of the exit shape $X^{\varepsilon}\left(\tau_{x}^{\varepsilon}, \cdot\right)$. Some results on exit time problems for stochastic partial differential equations can be found in $4,9,14,17,20,24,27,44$.

A large deviations principle characterizes the exponential decay rates of rare probabilities [20, 25, 28, 48]. An important step for characterizing 
Freidlin-Wentzell exit behaviors is to prove that solutions $X_{x}^{\varepsilon}$ to the system (1.1) satisfy a large deviations principle that is uniform with respect to the initial data $x \in D$. The exact definition of the uniform large deviations principle is Definition 3.1 .

In [13, Cerrai and Röckner proved that systems of stochastic reactiondiffusion equations with a small non-Gaussian noisy perturbation, like (1.1), satisfy a uniform large deviations principle that is uniform over subsets of initial data that are bounded in the supremum norm. Their result significantly improved upon large deviations results by Freidlin [27], Sowers [45], Peszat [39], and Kallianpur and Xiong [31] by removing assumptions about global Lipschitz continuity of the reaction terms and ellipticity assumptions about the multiplicative noise terms. Furthermore, [13] was the first paper to address the uniformity of the large deviations for the stochastic reactiondiffusion equation with respect to initial data in non-compact bounded sets.

This current paper significantly strengthens Cerrai and Röckner's results. First, we further relax Cerrai and Röckner's assumptions, removing their assumptions about the local Lipschitz continuity and polynomial growth rate of the reaction terms. We assume only that the reaction terms that can be written as the sum of a decreasing function and a Lipschitz continuous function (see Assumption 1). We prove that this large class of stochastic reaction-diffusion equations satisfy a large deviations principle that is uniform over sets of initial data that are bounded in the supremum norm (Theorem 4.1).

The other main results of this paper show that in two common situations the large deviations principle holds uniformly over all continuous initial data, not just over bounded subsets of initial data. These results enable the characterization of Freidlin-Wentzell exit time and exit shape asymptotics when the exit set $D$ is unbounded.

If the multiplicative noise coefficients $\sigma_{i n}$ are uniformly bounded, then the large deviations principle will hold uniformly over all continuous initial data (Theorem 4.2). In particular, whenever the system is exposed to additive noise, the large deviations principle holds uniformly over all initial data. Results about large deviations principles holding uniformly for unbounded sets of initial conditions, even in the additive noise case, were only previously known for equations with globally Lipschitz continuous reaction term [43.

Next, we consider the case where the reaction terms $f_{i}$ feature superlinear dissipativity. This means that there exist constants $\mu>0, m>1$, 
and $c_{0}>0$ such that the decreasing functions $v_{i} \mapsto g_{i}\left(t, \xi, v_{i}\right)$ satisfy

$$
g_{i}\left(t, \xi, v_{i}\right) \operatorname{sign}\left(v_{i}\right) \leq-\mu\left|v_{i}\right|^{m} \text { for }\left|v_{i}\right|>c_{0} .
$$

This case is motivated by the Allen-Cahn equation where the reaction term is an odd-degree polynomial with negative leading coefficient such as $f_{i}(t, \xi, x)=$ $-x_{i}^{3}+x_{i}$ (see, for example, 24]). Such a superlinear dissipative nonlinearity strongly forces the solutions towards finite values. In the presence of superlinear dissipativity, the large deviations principle can be uniform over all continuous initial conditions even when the multiplicative noise coefficients are unbounded (Theorem 4.3). The allowable growth rate of the multiplicative noise coefficients depends on the degree $m$ of super-linear dissipativity of the reaction term. This is the first result showing that the large deviations principle holds uniformly over all initial conditions when the multiplicative noise coefficients are unbounded.

Proving that these large deviations principles hold uniformly over unbounded sets of initial data requires a fundamentally different approach than the one used by Cerrai and Röckner [13]. Their argument relies on the assumption that the forcing term $f$ is locally Lipschitz continuous, and then uses localization techniques to approximate their equation by equations with globally Lipschitz forcing terms to prove the results. Such an approach can never lead to results that are uniform over unbounded sets of initial data.

The assumption of local Lipschitz continuity is replaced by a monotonicity condition (see, for example, [35]). In Assumption [1, below, we assume that $f_{i}=g_{i}+h_{i}$ is the sum of a decreasing function $g_{i}$, which does not need to be locally Lipschitz continuous, and a globally Lipschitz continuous function $h_{i}$. In Section 6 we prove that this assumption implies that an associated solution mapping is globally Lipschitz continuous, even when $g_{i}$ fails to be locally Lipschitz continuous. No localization techniques are required, enabling the proof of results that are uniform over unbounded subsets of initial data.

The proofs of the three main uniform large deviations results (Theorems 4.1, 4.2, and 4.3) are based on a variational principle for functions of infinite dimensional Wiener processes that is due to Budhiraja and Dupuis [5]. In the context of the reaction-diffusion equation (1.1), Budhiraja and Dupuis proved that for any bounded, continuous $h: C([0, T] \times \overline{\mathcal{O}} \times\{1, \ldots, r\}) \rightarrow \mathbb{R}$, $\varepsilon>0$, and $x \in C(\overline{\mathcal{O}} \times\{1, \ldots, r\})$,

$\varepsilon \log \mathbb{E} \exp \left(-\frac{h\left(X_{x}^{\varepsilon}\right)}{\varepsilon}\right)=-\inf _{u \in \mathscr{A}} \mathbb{E}\left[\frac{1}{2} \sum_{n=1}^{r} \int_{0}^{T} \int_{\mathcal{O}}\left|u_{n}(s, \xi)\right|^{2} d \xi d s+h\left(X_{x}^{\varepsilon, u}\right)\right]$. 
In the above expression, $\mathscr{A}$ is a collection of stochastic controls $u \in L^{2}(\Omega \times$ $[0, T] \times \mathcal{O} \times\{1, . ., r\})$ that are adapted to the natural filtration of the driving noises and $X_{x}^{\varepsilon, u}$ is the solution to the controlled reaction diffusion equation

$$
\left\{\begin{aligned}
& \frac{\partial X_{x, i}^{\varepsilon, u}}{\partial t}(t, \xi)=\mathcal{A}_{i} X_{x, i}^{\varepsilon, u}(t, \xi)+f_{i}\left(t, \xi, X_{x}^{\varepsilon, u}(t, \xi)\right) \\
&+\sqrt{\varepsilon} \sum_{n=1}^{r} \sigma_{i n}\left(t, \xi, X_{x}^{\varepsilon, u}(t, \xi)\right) \frac{\partial w_{n}}{\partial t}(t, \xi) \\
&+\sum_{n=1}^{r} \sigma_{i n}\left(t, \xi, X_{x}^{\varepsilon, u}(t, \xi)\right) Q_{n} u_{n}(t, \xi), \\
& X_{x}^{\varepsilon, u}(t, \xi)=0, \quad \xi \in \partial \mathcal{O}, \quad t \geq 0 \\
& X_{x}^{\varepsilon, u}(0, \xi)=x(\xi), \quad \xi \in \mathcal{O} .
\end{aligned}\right.
$$

The linear operators $Q_{n}$ are the covariances of the noises $w_{n}$ (see Assumption 4 below).

A major advancement in streamlining the proofs of uniform large deviations principles for small-noise SPDEs is the weak convergence approach due to Budhiraja, Dupuis, and Maroulas [7. In the context of these reaction diffusion equations, their result shows that $X_{x}^{\varepsilon}$ satisfy a large deviations principle that holds uniformly over compact sets of initial data if whenever $x_{n} \rightarrow x$ in the supremum norm, $\varepsilon_{n} \rightarrow 0$ and $u_{n} \rightarrow u$ in distribution in the weak topology on $L^{2}([0, T] \times \mathcal{O} \times\{1, \ldots, r\})$, the associated control problems $X_{x_{n}}^{\varepsilon_{n}, u_{n}}$ converge weakly to $X_{x}^{0, u}$. Many authors have applied this approach to prove that many SPDEs satisfy large deviations results that are uniform over compact sets of initial data [1,3, 6, 8, 9, 13, 18, 22, 26, 29, 30, 32, 34, 36, 37, 40 42, 46, 47, 49, 50]. The restriction to compact sets of initial data is due to the fact that their argument is based on weak convergence and if the initial data, $x_{n}$, do not belong to a compact subset then it is possible that no subsequence of $X_{x_{n}}^{\varepsilon_{n}, u_{n}}$ will converge weakly.

Of course, there are many applications, including Freidlin-Wentzell exit problems, where large deviations principles must hold uniformly over noncompact sets. In fact, because bounded subsets of infinite dimensional Banach spaces cannot be compact, Cerrai and Röckner's results about uniformity of large deviations principles holding over bounded subsets of initial data [13] cannot be proved directly via the weak convergence approach.

In [43, we proved that the variational principle can be used to prove large deviations principles that are uniform over non-compact and even unbounded subsets of initial data, but we require a stronger notion of convergence of controlled equations than weak convergence. Specifically, if for a 
set $D \subset C(\overline{\mathcal{O}} \times\{1, \ldots, r\})$ of continuous initial data and for any $\delta>0$ and $N>0$

$$
\lim _{\varepsilon \rightarrow 0} \sup _{x \in D} \sup _{u \in \mathscr{A}_{N}} \mathbb{P}\left(\left|X_{x}^{\varepsilon, u}-X_{x}^{0, u}\right|_{C([0, T] \times \overline{\mathcal{O}} \times\{1, \ldots, r\})}>\delta\right)=0,
$$

then $\left\{X_{x}^{\varepsilon}\right\}$ satisfies a uniform large deviations principle that is uniform over $x \in D$. In the above expression, $\mathscr{A}_{N}$ is the set of controls

$$
\mathscr{A}_{N}:=\left\{u \in \mathscr{A}: \mathbb{P}\left(\sum_{n=1}^{r} \int_{0}^{T} \int_{\mathcal{O}}\left|u_{n}(s, \xi)\right|^{2} d \xi d s \leq N\right)=1\right\} .
$$

In this paper, we are particularly interested in the case where $D=C(\overline{\mathcal{O}} \times$ $\{1, \ldots, r\})$ is the entire function space. Using the specific form of the systems of stochastic reaction diffusion equations (1.1), we will be able to prove this kind of convergence in probability holds uniformly over unbounded subsets of initial data, proving our main results.

In Section 2 we fix our main notations, present the main assumptions, and define the mild solution. In Section 3 we recall the definition of the uniform large deviations principle (ULDP) and we recall the major results about variational representations of infinite dimensional Brownian motion and sufficient conditions that imply uniform large deviations principles. In Section 4, we present the three main results of the paper. In Section 5, we give an example application of the main results.

Before proving the three main results, in Section 6 we study the properties of a fixed-point mapping $\mathcal{M}$ and show that this mapping is well-posed and globally Lipschitz continuous under our weak assumptions that the vector field $f$ is the sum of a decreasing function and a Lipschitz continuous function. In Section 7 we prove that the mild solutions to the stochastic reaction-diffusion equations and the controlled stochastic reaction diffusion equation exist and are unique under our weak assumptions. These existence and uniqueness results do not appear elsewhere in the literature.

In Section 8, we prove Theorem 4.1, which says that Cerrai and Röckner's 13. result about uniformity of the large deviations principle over bounded subsets can be recovered under our weaker assumptions. In Section 9, we prove Theorem 4.2, which says that when $\sigma$ is uniformly bounded, the large deviations principle is uniform over all initial conditions. In Section 10, we prove Theorem 4.3, which says that when the non-linearity $f$ features super-linear dissipativity and $\sigma$ is unbounded but does not grow too quickly, then the stochastic reaction-diffusion equation satisfies a large deviations principle that is uniform over all initial conditions. 
In Appendix $\mathrm{A}$ we recall results about the left-derivative of a supremum norm for a continuous process. In Appendix B we recall important estimates on the stochastic convolution due to Cerrai [11,12]. In Appendix C, we recall bounds that are uniform with respect to the initial conditions of a stochastic reaction-diffusion equation when the reaction terms features super-linear dissipativity.

\section{Notations and assumptions}

\subsection{Notations}

For a Euclidean set $A \subset \mathbb{R}^{j}$ for some $j \in \mathbb{N}$ let $C(A)$ be the set of continuous functions $y: A \rightarrow \mathbb{R}$. Because of the imposed boundary conditions in (1.1), we will work in the spaces of continuous functions with zero boundary conditions. Let

$$
\tilde{E}:=\{y \in C(\overline{\mathcal{O}}): y(\xi)=0 \text { for } \xi \in \partial \mathcal{O}\} .
$$

be the space of continuous functions on $\overline{\mathcal{O}}$ with zero boundary conditions endowed with the supremum norm

$$
|y|_{\tilde{E}}:=\sup _{\xi \in \mathcal{O}}|y(\xi)|
$$

Any continuous vector-valued function $x=\left(x_{1}, \ldots, x_{r}\right): \overline{\mathcal{O}} \rightarrow \mathbb{R}^{r}$ can be equivalently thought of as a scalar-valued continuous function in the space $C(\overline{\mathcal{O}} \times\{1, \ldots, r\})$. Let

$$
E:=\left\{x \in C(\overline{\mathcal{O}} \times\{1, \ldots, r\}): x_{i}(\xi)=0 \text { for } i \in\{1, \ldots r\}, \xi \in \partial \mathcal{O}\right\}
$$

and, for $T>0$,

$$
E_{T}:=\left\{\begin{array}{l}
\varphi \in C([0, T] \times \overline{\mathcal{O}} \times\{1, . ., r\}): \\
\varphi_{i}(t, \xi)=0 \text { for } i \in\{1, \ldots, r\}, t \in[0, T], \xi \in \partial \mathcal{O}
\end{array}\right\} .
$$

$E$ and $E_{T}$ are endowed with the supremum norms

$$
|x|_{E}:=\sup _{i \in\{1, \ldots, r\}} \sup _{\xi \in \mathcal{O}}\left|x_{i}(\xi)\right|
$$

and

$$
|\varphi|_{E_{T}}:=\sup _{i \in\{1, \ldots, r\}} \sup _{\xi \in \mathcal{O}} \sup _{t \in[0, T]}\left|\varphi_{i}(t, \xi)\right|
$$


We remark that this is a slightly different definition than the $E=C(\overline{\mathcal{O}}$ : $\left.\mathbb{R}^{r}\right)$ with $|x|_{E}=\sup _{\xi \in \overline{\mathcal{O}}}\left(\sum_{i=1}^{r}\left|x_{i}(\xi)\right|^{2}\right)^{\frac{1}{2}}$ norm that was used in [13]. Even though the norms are equivalent, the supremum norm is more convenient for our purposes than the mixture of the supremum and Euclidean norms.

We will show in Theorem 7.1 that the solutions $X^{\varepsilon}$ to the SPDE (1.1) exist, are unique, and are $E_{T}$-valued if their initial data is in $E$.

Throughout the paper we will use other common function spaces including $L^{p}$ spaces. If no measure is specified, then $L^{p}$ spaces are defined with respect to the Lebesgue measure on uncountable sets and the counting measure on discrete sets. For example for $p \geq 1, L^{p}([0, T] \times \mathcal{O} \times\{1, . . r\})$ is the set of functions $u:[0, T] \times \mathcal{O} \times\{1, \ldots, r\}$ for which the norm

$$
|u|_{L^{p}([0, T] \times \mathcal{O} \times\{1, \ldots, r\})}:=\left(\sum_{n=1}^{r} \int_{0}^{T} \int_{\mathcal{O}}\left|u_{n}(t, \xi)\right|^{p} d \xi d t\right)^{\frac{1}{p}}<+\infty .
$$

For any Banach spaces $\mathcal{E}_{1}, \mathcal{E}_{2}$, the set $\mathscr{L}\left(\mathcal{E}_{1}, \mathcal{E}_{2}\right)$ is the space of bounded linear operators from $\mathcal{E}_{1}$ to $\mathcal{E}_{2}$. If $\mathcal{E}_{1}=\mathcal{E}_{2}$, then the notation $\mathscr{L}\left(\mathcal{E}_{1}\right)=$ $\mathscr{L}\left(\mathcal{E}_{1}, \mathcal{E}_{1}\right)$.

\subsection{Main assumptions}

Now we specify our main assumptions about the objects in (1.1). Assumptions 1, 2, 3, and 4 hold throughout the paper. Later in Section 4 we introduce Assumption 5, which is only used in Theorem 4.2 and Assumption 6 . which is only used in Theorem 4.3 .

Assumption 1 (Vector field). The vector field $f:[0,+\infty) \times \mathcal{O} \times \mathbb{R}^{r} \times$ $\{1, \ldots, r\} \rightarrow \mathbb{R}$ can be written as

$$
f_{i}(t, \xi, u)=g_{i}\left(t, \xi, u_{i}\right)+h_{i}(t, \xi, u) .
$$

For any $i \in\{1, \ldots, r\}, t \geq 0, \xi \in \mathcal{O}$, the function $\mathbb{R} \ni x \mapsto g_{i}(t, \xi, x)$ is continuous and decreasing in the sense that for any $x, y \in \mathbb{R}$ such that $x>y$

$$
g_{i}(t, \xi, x)-g_{i}(t, \xi, y) \leq 0,
$$

There exists an increasing function $L:[0,+\infty) \rightarrow[0,+\infty)$ such that for any $x, y \in \mathbb{R}^{r}$,

$$
\sup _{i \in\{1, \ldots, r\}} \sup _{s \in[0, t]} \sup _{\xi \in \mathcal{O}}\left|h_{i}(s, \xi, x)-h_{i}(s, \xi, y)\right| \leq L(t) \sup _{i \in\{1, \ldots, r\}}\left|x_{i}-y_{i}\right|
$$


and

$$
\sup _{i \in\{1, \ldots, r\}} \sup _{s \in[0, t]} \sup _{\xi \in \mathcal{O}}\left|h_{i}(s, \xi, x)\right| \leq L(t)\left(1+\sup _{i \in\{1, \ldots, r\}}\left|x_{i}\right|\right) .
$$

Assumption 2 (Multiplicative noise coefficient). There exists an increasing function $L:[0,+\infty) \rightarrow[0,+\infty)$ such that for all $x, y \in \mathbb{R}^{r}$,

$$
\sup _{i, n \in\{1, \ldots, r\}} \sup _{s \in[0, t]} \sup _{\xi \in \mathcal{O}}\left|\sigma_{i n}(s, \xi, x)-\sigma_{i n}(s, \xi, y)\right| \leq L(t) \sup _{i \in\{1, \ldots, r\}}\left|x_{i}-y_{i}\right|
$$

and for any $x \in \mathbb{R}^{r}$

$$
\sup _{i, n \in\{1, \ldots, r\}} \sup _{s \in[0, t]} \sup _{\xi \in \mathcal{O}}\left|\sigma_{i n}(s, \xi, x)\right| \leq L(t)\left(1+\sup _{i \in\{1, \ldots, r\}}\left|x_{i}\right|\right)
$$

Assumption 3 (Elliptic operators). The spatial domain $\mathcal{O} \subset \mathbb{R}^{d}$ is open, bounded, and has smooth boundary. For $i \in\{1, \ldots, r\}$, the second-order elliptic operators $\mathcal{A}_{i}$ are of the form

$$
\mathcal{A}_{i} \varphi(\xi):=\sum_{j=1}^{d} \sum_{k=1}^{d} a_{j k}^{i}(\xi) \frac{\partial^{2} \varphi}{\partial \xi_{j} \partial \xi_{k}}(\xi)+\sum_{j=1}^{d} b_{j}^{i}(\xi) \frac{\partial \varphi}{\partial \xi_{j}}(\xi) .
$$

In the above expression, $a_{j k}^{i}: \overline{\mathcal{O}} \rightarrow \mathbb{R}$ are continuously differentiable and $b_{j}^{i}: \overline{\mathcal{O}} \rightarrow \mathbb{R}$ are continuous. The matrix $\left(a_{j k}^{i}(\xi)\right)_{j k}$ is symmetric and uniformly elliptic in the sense that there exists $\kappa>0$ such that for any vector $\left(x_{1}, \ldots, x_{d}\right)$,

$$
\inf _{\xi \in \mathcal{O}} \inf _{i \in\{1, \ldots, r\}} \sum_{j=1}^{d} \sum_{k=1}^{d} a_{j k}^{i}(\xi) x_{j} x_{k} \geq \kappa \sum_{j=1}^{d} x_{j}^{2} .
$$

Proposition 2.1. The operators $\mathcal{A}_{i}$ can be written as

$$
\mathcal{A}_{i}=\mathcal{B}_{i}+\mathcal{L}_{i}
$$

where

$$
\mathcal{B}_{i} \varphi(\xi):=\sum_{j=1}^{d} \sum_{k=1}^{d} \frac{\partial}{\partial \xi_{k}}\left(a_{j k}^{i}(\xi) \frac{\partial \varphi}{\partial \xi_{j}}(\xi)\right)
$$

is self-adjoint and

$$
\mathcal{L}_{i} \varphi(\xi):=\sum_{j=1}^{d}\left(b_{j}^{i}(\xi)-\sum_{k=1}^{d} \frac{\partial a_{j k}^{i}}{\partial \xi_{k}}(\xi)\right) \frac{\partial \varphi}{\partial \xi_{j}}(\xi)
$$


is a first-order differential operator.

For each $i \in\{1, \ldots, r\}$, there exists an orthonormal system of eigenvectors $\left\{e_{i, k}\right\}_{k=1}^{\infty} \subset L^{2}(\mathcal{O})$ and eigenvalues $\left\{\alpha_{i, k}\right\}_{k=1}^{\infty}$ such that the realization $B_{i}$ of $\mathcal{B}_{i}$ in $L^{2}(\mathcal{O})$ with the imposed boundary conditions satisfies

$$
B_{i} e_{i, k}=-\alpha_{i, k} e_{i, k}
$$

The eigenvalues are non-negative, diverge to infinity, and can be written in increasing order $0 \leq \alpha_{i, k} \leq \alpha_{i, k+1}$. By elliptic regularity results, for fixed $k, i, e_{i, k} \in \tilde{E}$ defined in (2.1). See, for example, [23, Chapter 6.5].

Assumption 4 (Noise). Fix a filtered probability space $\left(\Omega, \mathcal{F},\left\{\mathcal{F}_{t}\right\}, \mathbb{P}\right)$. The driving noise $w=\left(w_{1}, \ldots, w_{r}\right)$ can be formally written as the sum

$$
w_{n}(t, \xi):=\sum_{j=1}^{\infty} \lambda_{n, j} W_{n, j}(t) f_{n, j}(\xi)
$$

where for fixed $n \in\{1, \ldots, r\},\left\{f_{n, j}\right\}_{j=1}^{\infty}$ is an orthonormal basis of $L^{2}(\mathcal{O})$, and for each fixed $n, j f_{n, j} \in \tilde{E}$. Such a sequence $f_{n, j}$ exists because one could take $f_{n, j}:=e_{n, j}$ (2.18). $\quad\left\{\left\{W_{n, j}\right\}_{j=1}^{\infty}\right\}_{n=1}^{r}$ is a countable collection of independent one-dimensional Brownian motions on $\left(\Omega, \mathcal{F},\left\{\mathcal{F}_{t}\right\}, \mathbb{P}\right)$. The numbers $\lambda_{n, j} \geq 0$ and there exists $\beta \in(0,1)$ and $\rho \in[2,+\infty]$ such that

$$
\begin{gathered}
\frac{\beta(\rho-2)}{\rho}<1, \\
\sum_{i=1}^{r} \sum_{k=1}^{\infty} \alpha_{i, k}^{-\beta}\left|e_{i, k}\right|_{\tilde{E}}^{2}<\infty,
\end{gathered}
$$

and

$$
\begin{aligned}
& \sum_{j=1}^{\infty} \sum_{n=1}^{r} \lambda_{n, j}^{\rho}\left|f_{n, j}\right|_{\tilde{E}}^{2}<\infty, \text { if } \rho<+\infty \\
& \text { or } \sup _{j} \sup _{n} \lambda_{n, j}<+\infty, \text { if } \rho=+\infty,
\end{aligned}
$$

where $\alpha_{i, k}, e_{i, k}$ are the eigenvalues of $B_{i}$ from (2.18).

Remark 2.2. Cerrai [11,12] proved that Assumption4 is a sufficient condition that implies that mild solutions to the stochastic reaction-diffusion equation are continuous functions of space and time. 
For $n \in\{1, . . r\}$, let $Q_{n}: L^{2}(\mathcal{O}) \rightarrow L^{2}(\mathcal{O})$ be the bounded linear operator

$$
Q_{n} f_{n, j}=\lambda_{n, j} f_{n, j}
$$

and let $Q: L^{2}(\mathcal{O} \times\{1, \ldots r\}) \rightarrow L^{2}(\mathcal{O} \times\{1, \ldots r\})$ be defined so that for any $n \in\{1, \ldots, r\}$, and $\xi \in \mathcal{O}$,

$$
[Q u]_{n}(\xi)=\left[Q_{n} u_{n}\right](\xi)
$$

\subsection{Semigroups and mild solution}

Let $S_{i}(t)$ be the semigroup on $\tilde{E}(2.1)$ generated by the elliptic operator $\mathcal{A}_{i}$ with zero boundary conditions. It is standard that $S_{i}(t)$ is a $C_{0}$ semigroup on $\tilde{E}$ (see [23]).

For $x \in E$, let $[S(t) x]_{i}(\xi):=\left[S_{i}(t) x_{i}\right](\xi)$. In this way, $S(t): E \rightarrow E$ is a $C_{0}$ contraction semigroup on $E$.

The mild solution for $X_{x, i}^{\varepsilon}$ is defined to be the solution to the system of integral equations for $i \in\{1, \ldots r\}$,

$$
\begin{aligned}
X_{x, i}^{\varepsilon}(t)= & S_{i}(t) x_{i}+\int_{0}^{t} S_{i}(t-s) F_{i}\left(s, X_{x}^{\varepsilon}(s)\right) d s \\
& +\sqrt{\varepsilon} \sum_{n=1}^{r} \int_{0}^{t} S_{i}(t-s) R_{i n}\left(s, X_{x}^{\varepsilon}(s)\right) d w_{n}(s) .
\end{aligned}
$$

In the above equation, the spatial variable $\xi$ has been suppressed.

$F_{i}:[0,+\infty) \times E \rightarrow \tilde{E}$ is the Nemytskii operator where for any $i \in\{1, \ldots r\}$, $t>0, \xi \in \mathcal{O}$, and $x \in E$,

$$
\left[F_{i}(t, x)\right](\xi):=f_{i}(t, \xi, x(\xi)),
$$

and $R_{\text {in }}:[0,+\infty) \times E \rightarrow \mathscr{L}\left(L^{2}(\mathcal{O})\right)$ is defined such that for any $i, n \in$ $\{1, . . r\}, t>0, \xi \in \mathcal{O}$, and $x \in E$, and $h \in L^{2}(\mathcal{O})$,

$$
\left[R_{\text {in }}(t, x) h\right](\xi)=\sigma_{i n}(t, \xi, x(\xi)) h(\xi) .
$$

By the definition of the noise (2.19), the stochastic convolution can be understood as the infinite sum of one-dimensional Ito integrals

$$
\int_{0}^{t} S_{i}(t-s) R_{i n}\left(s, X_{x}^{\varepsilon}(s)\right) d w_{n}(s)=\sum_{j=1}^{\infty} \int_{0}^{t} S_{i}(t-s) R_{i n}\left(s, X_{x}^{\varepsilon}(s)\right) \lambda_{n, j} f_{n, j} d W_{n, j}(s) .
$$

The properties of the stochastic convolution can be found in [11] and are included in Appendix B below. 
Definition 2.3. The mild solution to (1.1) is defined to be the $E_{T}$-valued solution to

$$
X_{x}^{\varepsilon}(t)=S(t) x+\int_{0}^{t} S(t-s) F\left(s, X_{x}^{\varepsilon}(s)\right) d s+\sqrt{\varepsilon} \int_{0}^{t} S(t-s) R\left(s, X_{x}^{\varepsilon}(s)\right) d w(s)
$$

In the above equation $F:[0,+\infty) \times E \rightarrow E$ is the vector $F=\left(F_{1}, \ldots, F_{r}\right)$ and $R:[0,+\infty) \times E \rightarrow \mathscr{L}\left(L^{2}(\mathcal{O} \times\{1, \ldots, r\})\right)$ is the matrix $R=\left(R_{\text {in }}\right)_{\text {in }}$. $w=\left(w_{1}, \ldots w_{r}\right)$. We prove that there exists a unique mild solution in Section [7.

To prove the large deviations results we will study the convergence properties of mild solutions to the stochastic control problems (1.6). The mild solution to (1.6) will solve the integral equation

$$
\begin{aligned}
X_{x}^{\varepsilon, u}(t)= & S(t) x+\int_{0}^{t} S(t-s) F\left(s, X_{x}^{\varepsilon, u}(s)\right) d s \\
& +\sqrt{\varepsilon} \int_{0}^{t} S(t-s) R\left(s, X_{x}^{\varepsilon, u}(s)\right) d w(s) \\
& +\int_{0}^{t} S(t-s) R\left(s, X_{x}^{\varepsilon, u}(s)\right) Q u(s) d s .
\end{aligned}
$$

\section{Uniform large deviations principle and the equicon- tinuous uniform Laplace principle}

In this section we recall the definitions of Freidlin and Wentzell's uniform large deviations principle (ULDP) and a result from [43] that proves that the uniform convergence in probability of certain controlled process implies that a collection of processes satisfies the ULDP.

Let $(\mathcal{E}, \vartheta)$ be a Polish space and let $\mathcal{E}_{0}$ be a set used for indexing (there are no topological assumptions on $\mathcal{E}_{0}$ ). When we apply these results in the sequel, we will set $\mathcal{E}=E_{T}$ and $\mathcal{E}_{0}=E$. Let $\left\{Y_{x}^{\varepsilon}\right\}_{x \in \mathcal{E}_{0}, \varepsilon>0}$ be a collection of $\mathcal{E}$-valued random variables. For every $x \in \mathcal{E}_{0}$, let $I_{x}: \mathcal{E} \rightarrow[0,+\infty]$ be a lower-semicontinuous function called a rate function. For each $x \in \mathcal{E}_{0}$ and $s \geq 0$, let

$$
\Phi_{x}(s):=\left\{\varphi \in \mathcal{E}: I_{x}(\varphi) \leq s\right\}
$$

be the level sets of the rate function. Let $\operatorname{dist}_{\mathcal{E}}: \mathcal{E} \times 2^{\mathcal{E}} \rightarrow[0,+\infty)$ be defined as the minimal distance between an element of $\mathcal{E}$ and a set

$$
\operatorname{dist}_{\mathcal{E}}(\varphi, \Psi):=\inf _{\psi \in \Psi} \vartheta(\varphi, \psi)
$$


Definition 3.1 (Uniform large deviations principle (ULDP) (Section 3.3 of [28])). A family $\left\{Y_{x}^{\varepsilon}\right\}_{x \in \mathcal{E}_{0}, \varepsilon>0}$ of $\mathcal{E}$-valued random variables satisfies a uniform large deviations principle uniformly over a set $D \subset \mathcal{E}_{0}$ with respect to the rate functions $I_{x}$ if

1. for any $\delta>0$ and $s_{0} \geq 0$,

$$
\liminf _{\varepsilon \rightarrow 0} \inf _{x \in D} \inf _{\varphi \in \Phi_{x}\left(s_{0}\right)}\left(\varepsilon \log \mathbb{P}\left(\vartheta\left(Y_{x}^{\varepsilon}, \varphi\right)<\delta\right)+I_{x}(\varphi)\right) \geq 0 .
$$

and

2. for any $\delta>0$ and $s_{0} \geq 0$,

$$
\limsup _{\varepsilon \rightarrow 0} \sup _{x \in D} \sup _{s \in\left[0, s_{0}\right]}\left(\varepsilon \log \mathbb{P}\left(\operatorname{dist}_{\mathcal{E}}\left(Y_{x}^{\varepsilon}, \Phi_{x}(s)\right) \geq \delta\right)+s\right) \leq 0 .
$$

The following theorem describes a sufficient condition that implies that measurable functions of a countable collection of Brownian motions satisfy the ULDP.

Suppose that $W=\left\{W_{j}(\cdot)\right\}_{j=1}^{\infty}$ is a countable collection of i.i.d. onedimensional Brownian motions on a filtered probability space $\left(\Omega, \mathcal{F},\left\{\mathcal{F}_{t}\right\}, \mathbb{P}\right)$. For fixed $T>0$ and any $x \in \mathcal{E}_{0}$ suppose that $\mathscr{G}_{x}: C([0, T] \times \mathbb{N}) \rightarrow \mathcal{E}$ is a measurable mapping. For $\varepsilon \geq 0$ and $x \in \mathcal{E}_{0}$, let

$$
Y_{x}^{\varepsilon}:=\mathcal{G}_{x}(\sqrt{\varepsilon} W)
$$

For each $N>0$, let $\mathscr{B}_{N}$ be the collection of $u \in L^{2}(\Omega \times[0, T] \times \mathbb{N})$ that are adapted to the filtration $\mathcal{F}_{t}$ and satisfy

$$
\mathbb{P}\left(\sum_{j=1}^{\infty} \int_{0}^{T}\left|u_{j}(s)\right|^{2} d s \leq N\right)=1 .
$$

For each $u \in \mathscr{B}_{N}$, let $Y_{x}^{\varepsilon, u}$ denote the controlled $\mathcal{E}$-valued random variable

$$
Y_{x}^{\varepsilon, u}:=\mathcal{G}_{x}\left(\sqrt{\varepsilon} W+\int_{0}^{\cdot} u(s) d s\right) .
$$

Proving that a family $Y_{x}^{\varepsilon}$ satisfies a ULDP directly using Definition 3.1 can be cumbersome. The proofs of the main results in this paper are based on Theorem 2.13 of [43], which proves that uniform convergence in probability of the controlled system $Y_{x}^{\varepsilon, u}$ to $Y_{x}^{0, u}$ as $\varepsilon \rightarrow 0$ implies the ULDP. 
Theorem 3.2 (Theorem 2.13 of [43]). Let $D \subset \mathcal{E}_{0}$. If for any $\delta>0$ and $N>0$,

$$
\lim _{\varepsilon \rightarrow 0} \sup _{x \in D} \sup _{u \in \mathscr{B}_{N}} \mathbb{P}\left(\vartheta\left(Y_{x}^{\varepsilon, u}, Y_{x}^{0, u}\right)>\delta\right)=0,
$$

then the family $\left\{Y_{x}^{\varepsilon}\right\}$ satisfies a ULDP uniformly over $D$ with respect to the rate functions $I_{x}: \mathcal{E} \rightarrow[0,+\infty]$ defined by

$$
I_{x}(\varphi):=\inf \left\{\frac{1}{2} \sum_{j=1}^{\infty} \int_{0}^{T}\left|u_{j}(s)\right|^{2} d s: \varphi=Y_{x}^{0, u}, u \in L^{2}([0, T] \times \mathbb{N})\right\} .
$$

In the context of the system of reaction-diffusion equations (2.29), we will let $\mathcal{E}_{0}:=E$ defined in (2.3) be the set of initial data and for a fixed time horizon $T>0$ let $\mathcal{E}=E_{T}$ defined in (2.4). Because the driving noise is defined in terms of a countable collection of i.i.d. Brownian motions $W=$ $\left\{\left\{W_{n, j}\right\}_{j=1}^{\infty}\right\}_{n=1}^{r}$ (see (2.19)) and because we will show that the mild solutions (2.29) exist and are unique (see Corollary (7.2), there exists a measurable mapping $\mathscr{G}_{x}: C([0, T] \times\{1, \ldots, r\} \times \mathbb{N}) \rightarrow \mathcal{E}$ such that $X_{x}^{\varepsilon}:=\mathscr{G}_{x}(\sqrt{\varepsilon} W)$ solves (2.29).

According to (3.5), the spaces $\mathscr{B}_{N}$ will consist of adapted processes $u \in$ $L^{2}(\Omega \times[0, T] \times\{1, \ldots, r\} \times \mathbb{N})$ satisfying

$$
\mathbb{P}\left(\sum_{j=1}^{\infty} \sum_{n=1}^{r} \int_{0}^{T}\left|u_{n, j}(s)\right|^{2} d s \leq N\right)=1
$$

For $N>0$ and $u \in \mathscr{B}_{N}$, the controlled processes $Y_{x}^{\varepsilon, u}=\mathscr{G}_{x}\left(\sqrt{\varepsilon} W+\int_{0}^{\cdot} u(s) d s\right)$ solves the integral equation

$$
\begin{aligned}
Y_{x}^{\varepsilon, u}(t)= & S(t) x+\int_{0}^{t} S(t-s) F\left(s, Y_{x}^{\varepsilon, u}(s)\right) d s \\
& +\sqrt{\varepsilon} \int_{0}^{t} S(t-s) R\left(s, Y_{x}^{\varepsilon, u}(s)\right) d w(s) \\
& +\int_{0}^{t} S(t-s) R\left(s, Y_{x}^{\varepsilon, u}(s)\right) Q \mathcal{I} u(s) d s
\end{aligned}
$$

where $\mathcal{I}: L^{2}(\{1, \ldots, r\} \times \mathbb{N}) \rightarrow L^{2}(\mathcal{O} \times\{1, \ldots, r\})$ is the isometry defined by

$$
[\mathcal{I} u]_{n}(\xi):=\sum_{j=1}^{\infty} u_{n, j} f_{n, j}(\xi)
$$


In the above expression, $f_{n, j}$ are the orthonormal basis defined in Assumption 4. The noise $w(t)$ is defined in Assumption 4. $F$ and $R$ are the Nemytskii operators defined in (2.27) and (2.28).

Because $\mathcal{I}$ is an isometry, we can equivalently define $\mathscr{A}_{N}:=\mathcal{I}\left(\mathscr{B}_{N}\right)$ to be the family of adapted $L^{2}([0, T] \times \mathcal{O} \times\{1, \ldots r\})$ processes satisfying

$$
\mathbb{P}\left(\sum_{n=1}^{r} \int_{0}^{T} \int_{\mathcal{O}}\left|u_{n}(s, \xi)\right|^{2} d \xi d s \leq N\right)=1 .
$$

and then define $X_{x}^{\varepsilon, u}$ for $u \in \mathscr{A}_{N}$,

$$
\begin{aligned}
X_{x}^{\varepsilon, u}(t)= & S(t) x+\int_{0}^{t} S(t-s) F\left(s, X_{x}^{\varepsilon, u}(s)\right) d s \\
& +\sqrt{\varepsilon} \int_{0}^{t} S(t-s) R\left(s, X_{x}^{\varepsilon, u}(s)\right) d w(s) \\
& +\int_{0}^{t} S(t-s) R\left(s, X_{x}^{\varepsilon, u}(s)\right) Q u(s) d s .
\end{aligned}
$$

this agrees with (2.30).

The isometry between $\mathscr{B}_{N}$ and $\mathscr{A}_{\mathscr{N}}$ and Theorem 3.2 imply the following result that we will use to prove our three main results.

Corollary 3.3. Let $D$ be a subset of $E$. If for $T>0$ and any $\delta>0$, and $N>0$,

$$
\lim _{\varepsilon \rightarrow 0} \sup _{x \in D} \sup _{u \in \mathscr{B}_{N}} \mathbb{P}\left(\left|X_{x}^{\varepsilon, u}-X_{x}^{0, u}\right|_{E_{T}}>\delta\right)=0
$$

then the family $\left\{X_{x}^{\varepsilon}\right\}$ satisfies a ULDP in the $E_{T}$ norm uniformly over $D$ with respect to the rate functions $I_{x, T}: \mathcal{E} \rightarrow[0,+\infty]$ defined by

$$
I_{x, T}(\varphi):=\inf \left\{\frac{1}{2} \sum_{n=1}^{r} \int_{0}^{T} \int_{\mathcal{O}}\left|u_{n}(s, \xi)\right|^{2} d \xi d s: \varphi=X_{x}^{0, u}\right\}
$$

where the infimum is taken over all $u \in L^{2}([0, T] \times \mathcal{O} \times\{1, \ldots, r\})$.

\section{Main results}

The first main result of this paper proves that under Assumptions 1, 2, 3, and 4. the mild solutions $X_{x}^{\varepsilon}$ satisfy a large deviations principle that is uniform over bounded subsets of initial data $x$. This result generalizes the result of Cerrai and Röckner [13] by removing the restrictions to locally Lipschitz 
continuity and polynomial growth of the reaction term $f$. For these results, recall Definition 3.1 of the ULDP and the definitions of the rate function $I_{x, T}(3.14)$ and define the level sets for $s \geq 0$,

$$
\Phi_{x, T}(s):=\left\{\varphi \in E_{T}: I_{x, T}(\varphi) \leq s\right\} .
$$

Theorem 4.1. Assume Assumptions 1, 2, 3, and 4. For any fixed $T>0$, $X_{x}^{\varepsilon}$ satisfy a large deviations principle in $E_{T}$ that is uniform over bounded subsets of initial data. In particular, for any $K>0$, any $\delta>0$, and any $s_{0} \geq 0$,

$$
\liminf _{\varepsilon \rightarrow 0} \inf _{|x|_{E} \leq K} \inf _{\varphi \in \Phi_{x, T}\left(s_{0}\right)}\left(\varepsilon \log \mathbb{P}\left(\left|X_{x}^{\varepsilon}-\varphi\right|_{E_{T}}<\delta\right)+I_{x, T}(\varphi)\right) \geq 0
$$

and

$$
\limsup _{\varepsilon \rightarrow 0} \sup _{|x|_{E} \leq K} \sup _{s \in\left[0, s_{0}\right]}\left(\varepsilon \log \mathbb{P}\left(\operatorname{dist}_{E_{T}}\left(X_{x}^{\varepsilon}, \Phi_{x, T}(s)\right) \geq \delta\right)+s\right) \leq 0 .
$$

The proof of Theorem 4.1 is in Section 8 ,

The next result shows that if we restrict the multiplicative noise coefficients $\sigma_{i n}$ to be uniformly bounded, then the large deviations principle actually holds uniformly over unbounded subsets of initial data. We continue to assume Assumptions 11, 3, and 4 and we add the following strengthening of Assumption 2,

Assumption 5 (Bounded $\sigma$ ). There exists an increasing function $L:[0,+\infty) \rightarrow$ $[0,+\infty)$ such that for all $x, y \in \mathbb{R}^{r}$,

$$
\sup _{i, j \in\{1, \ldots, r\}} \sup _{s \in[0, t]} \sup _{\xi \in \mathcal{O}}\left|\sigma_{i j}(s, \xi, x)-\sigma_{i j}(s, \xi, y)\right| \leq L(t) \sup _{i \in\{1, \ldots, r\}}\left|x_{i}-y_{i}\right|
$$

and

$$
\sup _{i, j \in\{1, \ldots, r\}} \sup _{s \in[0, t]} \sup _{\xi \in \mathcal{O}} \sup _{x \in \mathbb{R}^{r}}\left|\sigma_{i j}(s, \xi, x)\right| \leq L(t) .
$$

Theorem 4.2. Assume Assumptions 1, 3, 4, and 5. For any fixed $T>0$, $X_{x}^{\varepsilon}$ satisfy a large deviations principle in $E_{T}$ that is uniform over all initial conditions in $E$. In particular, for any $\delta>0, s_{0} \geq 0$,

$$
\liminf _{\varepsilon \rightarrow 0} \inf _{x \in E} \inf _{\varphi \in \Phi_{x}\left(s_{0}\right)}\left(\varepsilon \log \mathbb{P}\left(\left|X_{x}^{\varepsilon}-\varphi\right|_{E_{T}}<\delta\right)+I_{x}(\varphi)\right) \geq 0,
$$

and

$$
\limsup _{\varepsilon \rightarrow 0} \sup _{x \in E} \sup _{s \in\left[0, s_{0}\right]}\left(\varepsilon \log \mathbb{P}\left(\operatorname{dist}_{E_{T}}\left(X_{x}^{\varepsilon}, \Phi_{x}(s)\right) \geq \delta\right)+s\right) \leq 0 .
$$


The proof of Theorem 4.2 is in Section 9.

The third main result identifies a sufficient condition that implies that the large deviations principle holds uniformly over all initial data even when $\sigma$ is unbounded. The result requires the reaction term $f$ to feature sufficiently strong superlinearly dissipativity to counteract the expansive effects of the unbounded $\sigma$. Specifically we assume the following.

Assumption 6 (Super-linear dissipativity). The reaction term $f$ can be written as $f_{i}=g_{i}+h_{i}$ where $g_{i}$ and $h_{i}$ satisfy Assumption 1. Additionally, there exists $m>1$ (not necessarily an integer), $\mu>0$, and $c_{0}>0$ such that for any $i \in\{1, \ldots, r\}, t>0, \xi \in \overline{\mathcal{O}}$, and $\left|v_{i}\right|>c_{0}$,

$$
g_{i}\left(t, \xi, v_{i}\right) \operatorname{sign}\left(v_{i}\right) \leq-\mu\left|v_{i}\right|^{m} .
$$

We further assume that there exists an increasing function $L:[0,+\infty) \rightarrow$ $[0,+\infty)$ such that for any $x, y \in \mathbb{R}^{r}$,

$$
\sup _{i, j \in\{1, \ldots, r\}} \sup _{s \in[0, t]} \sup _{\xi \in \mathcal{O}}\left|\sigma_{i j}(s, \xi, x)-\sigma_{i j}(s, \xi, y)\right| \leq L(t) \sup _{i \in\{1, \ldots, r\}}\left|x_{i}-y_{i}\right| .
$$

and that there exists

$$
\nu \in\left[0, \frac{m-1}{2}\left(1-\frac{\beta(\rho-2)}{\rho}\right)\right) \cap[0,1]
$$

such that for any $x \in \mathbb{R}^{r}$,

$$
\sup _{i, n \in\{1, \ldots, r\}} \sup _{\xi \in \mathcal{O}} \sup _{t \in[0, T]}\left|\sigma_{i n}(t, \xi, x)\right| \leq L(T)\left(1+\sup _{i \in\{1, \ldots, r\}}\left|x_{i}\right|\right)^{\nu} .
$$

Theorem 4.3. Assume Assumptions 1, 3, 4, and 6. For any fixed $T>0$ $X_{x}^{\varepsilon}$ satisfy a large deviations principle in $E_{T}$ that is uniform over all initial conditions in $E$. In particular, for any $\delta>0, s_{0} \geq 0$,

$$
\begin{gathered}
\liminf _{\varepsilon \rightarrow 0} \inf _{x \in E} \inf _{\varphi \in \Phi_{x}\left(s_{0}\right)}\left(\varepsilon \log \mathbb{P}\left(\left|X_{x}^{\varepsilon}-\varphi\right|_{E_{T}}<\delta\right)+I_{x, T}(\varphi)\right) \geq 0, \\
\limsup \sup _{\varepsilon \in 0} \sup _{x \in\left[0, s_{0}\right]}\left(\varepsilon \log \mathbb{P}\left(\operatorname{dist}_{E_{T}}\left(X_{x}^{\varepsilon}, \Phi_{x, T}(s)\right) \geq \delta\right)+s\right) \leq 0 .
\end{gathered}
$$

The proof of Theorem 4.3 is in Section 10. 


\section{Example: System of stochastic reaction-diffusion equations exposed to space-time white noise in spatial dimension $d=1$}

We consider a class of reaction-diffusion equations with polynomially dissipative forcing and polynomially growing multiplicative noise term in spatial dimension $d=1$. For simplicity, we only consider one equation $(r=1)$, rather than a system of equations. Let $m \geq 0$ and $\nu \leq 1$. $m$ does not need to be an integer. Let $X_{x}^{\varepsilon}(t, \xi)$ be the mild solution to

$$
\left\{\begin{aligned}
& \frac{\partial}{\partial t} X_{x}^{\varepsilon}(t, \xi)=\frac{\partial^{2}}{\partial \xi^{2}} X_{x}^{\varepsilon}(t, \xi)-\left|X_{x}^{\varepsilon}(t, \xi)\right|^{m} \operatorname{sign}\left(X_{x}^{\varepsilon}(t, \xi)\right) \\
& \quad+\sqrt{\varepsilon}\left(1+\left|X_{x}^{\varepsilon}(t, \xi)\right|\right)^{\nu} \frac{\partial w}{\partial t}(t, \xi) \\
& X_{x}^{\varepsilon}(0, \xi)=x(\xi) \\
& X_{x}^{\varepsilon}(t, 0)=X_{x}^{\varepsilon}(t, \pi)=0
\end{aligned}\right.
$$

defined on the one-dimensional spatial domain $\mathcal{O}=(0, \pi)$. $\frac{\partial w}{\partial t}$ is a spacetime white noise.

In this spatial dimension $d=1$ setting, the eigenvalues of $\frac{\partial^{2}}{\partial \xi^{2}}$ are $-\alpha_{k}$ where $\alpha_{k}=k^{2}$. Because $\frac{\partial w}{\partial t}$ is a space-time white noise, $\lambda_{j} \equiv 1$. These sequences satisfy Assumption 4 with $\rho=+\infty$ and any $\beta \in\left(\frac{1}{2}, 1\right)$.

For any $m \geq 0$, the function $g(x)=-|x|^{m} \operatorname{sign}(x)$ is decreasing. For any $\nu \leq 1, \sigma(x):=(1+|x|)^{\nu}$ is Lipschitz continuous. Therefore, if $m \geq 0$ and $\nu \leq 1$, Theorem 4.1 guarantees that $X_{x}^{\varepsilon}$ satisfies a ULDP that is uniform over bounded subsets of initial data.

If $\nu \leq 0$ (the case of bounded noise coefficients) and $m \geq 0$, then Theorem 4.2 guarantees that the system satisfies a uniform large deviations principle that is uniform over all $E$-valued initial data.

When $\nu$ satisfies

$$
\nu<\frac{(m-1)(1-\beta)}{2}<\frac{m-1}{4} \quad \text { and } \quad \nu \leq 1,
$$

Theorem 4.3 guarantees that $\left\{X_{x}^{\varepsilon}\right\}$ will satisfy a large deviations principle that is uniform over all $E$-valued initial data. The restriction $\nu \leq 1$ is required because $\sigma(x)=(1+|x|)^{\nu}$ fails to be globally Lipschitz continuous if $\nu>1$.

If $m=3$ and $\nu<\frac{1}{2}$, then Theorem 4.3 guarantees that $X_{x}^{\varepsilon}$ will satisfy a uniform large deviations principle that is uniform over all continuous initial data. If $m=5$ and $\nu<1$, then the large deviations principle will hold uniformly over all $E$-valued data. If $m>5$ and $\nu \leq 1$ then $X_{x}^{\varepsilon}$ will satisfy 
a uniform large deviations principle that is uniform over all $E$-valued initial conditions.

\section{Lipschitz continuity of $\mathcal{M}$}

In order to prove Theorems 4.1, 4.2, and 4.3, and even to prove that the mild solutions to (2.29) and (2.30) are well defined, we define a mapping $\mathcal{M}: E_{T} \rightarrow E_{T}$ that sends an element $z \in E_{T}$ to the fixed point solution

$$
\mathcal{M}(z)(t):=\int_{0}^{t} S(t-s) F(s, \mathcal{M}(z)(s)) d s+z(t) .
$$

In this section we prove that $\mathcal{M}$ is well-defined and globally Lipschitz continuous whenever $f$ satisfies Assumption 1, even if $f$ fails to be locally Lipschitz continuous.

The mapping $\mathcal{M}$ is essential to our investigation of the mild solutions to the reaction-diffusion equations because $X_{x}^{\varepsilon}$ will be a mild solution solving (2.29) if and only if

$$
X_{x}^{\varepsilon}=\mathcal{M}\left(S(\cdot) x+\sqrt{\varepsilon} Z_{x}^{\varepsilon}\right)
$$

where

$$
Z_{x}^{\varepsilon}(t)=\int_{0}^{t} S(t-s) R\left(s, X_{x}^{\varepsilon}(s)\right) d w(s)
$$

Similarly, $X_{x}^{\varepsilon, u}$ solves (2.30) if and only if

$$
X_{x}^{\varepsilon, u}=\mathcal{M}\left(S(\cdot) x+Y_{x}^{\varepsilon, u}+\sqrt{\varepsilon} Z_{x}^{\varepsilon, u}\right)
$$

where

$$
Y_{x}^{\varepsilon, u}(t)=\int_{0}^{t} S(t-s) R\left(s, X_{x}^{\varepsilon, u}(s)\right) Q u(s) d s
$$

and

$$
Z_{x}^{\varepsilon, u}(t)=\int_{0}^{t} S(t-s) R\left(s, X_{x}^{\varepsilon, u}(s)\right) d w(s) .
$$

Theorem 6.1. For any $z \in E_{T}$, there exists a solution $\mathcal{M}(z) \in E_{T}$ to (6.1).

Proof. Let $g_{i}$ be the non-increasing functions from Assumption 1, For $n \in \mathbb{N}$, $t \geq 0$ and $\xi \in \mathcal{O}$ define $x \mapsto g_{i, n}(t, \xi, x)$ to be the Yosida approximation

$$
g_{i, n}(t, \xi, x):=n\left(J_{i, n}(t, \xi, x)-x\right), \quad J_{i, n}(t, \xi, x)=\left(\bullet-\frac{1}{n} g_{i}(t, \xi, \bullet)\right)^{-1}(x) .
$$

Let $f_{i, n}(t, \xi, x):=g_{i, n}\left(t, \xi, x_{i}\right)+h_{i}(t, \xi, x)$. 
According to [19, Proposition D.11], for $t \geq 0, \xi \in \overline{\mathcal{O}}, i \in\{1, \ldots, r\}$, and $x, y \in \mathbb{R}$

$$
\begin{aligned}
& \left|g_{i, n}(t, \xi, x)-g_{i, n}(t, \xi, y)\right| \leq 2 n|x-y| \\
& \left|g_{i, n}(t, \xi, x)\right| \leq\left|g_{i}(t, \xi, x)\right| \\
& g_{i, n}(t, \xi, x)-g_{i, n}(t, \xi, y) \leq 0 \text { for } x>y \\
& \quad \lim _{n \rightarrow+\infty} g_{i, n}(t, \xi, x)=g_{i}(t, \xi, x)
\end{aligned}
$$

Because $h_{i}$ is Lipschitz continuous (2.10) and $f_{i, n}=g_{i, n}+h_{i}$, for any $t \geq 0, \xi \in \overline{\mathcal{O}}, i \in\{1, \ldots, r\}$, and $x, y \in \mathbb{R}^{r}$,

$$
\begin{aligned}
& \left|f_{i, n}(t, \xi, x)-f_{i, n}(t, \xi, y)\right| \leq(2 n+L(t))|x-y| \\
& \left|f_{i, n}(t, \xi, x)\right| \leq\left|f_{i}(t, \xi, x)\right|+2\left|h_{i}(t, \xi, x)\right| \\
& f_{i, n}(t, \xi, x)-f_{i, n}(t, \xi, y) \leq L(t)|x-y| \text { for } x>y, \\
& \quad \lim _{n \rightarrow+\infty} f_{i, n}(t, \xi, x)=f_{i}(t, \xi, x) .
\end{aligned}
$$

Let $F_{n}:[0, T] \times E \rightarrow E$ be the Nemytskii operator for $\left(f_{1, n}, \ldots, f_{r, n}\right)$

$$
\left[F_{n}(t, x)\right]_{i}(\xi)=f_{i, n}(t, \xi, x(\xi)) .
$$

Because the $f_{i, n}$ are each globally Lipschitz continuous, standard Picard iteration arguments show that there exists a unique solution $u_{n} \in E_{T}$ solving

$$
u_{n}(t)=\int_{0}^{t} S(t-s) F_{n}\left(s, u_{n}(s)\right) d s+z(t) .
$$

We prove some uniform bounds on the sequence $u_{n}$. Let $v_{n}(t)=u_{n}(t)-$ $z(t)$. These $v_{n}$ are weakly differentiable and they solve the integral equation

$$
v_{n}(t)=\int_{0}^{t} S(t-s) F_{n}\left(s, v_{n}(s)+z(s)\right) d s .
$$

The $v_{n}$ weakly solve the differential equation

$\frac{\partial v_{n, i}}{\partial t}(t, \xi)=\mathcal{A}_{i} v_{n, i}(t, \xi)+g_{i, n}\left(t, \xi, v_{n, i}(t, \xi)+z_{i}(t, \xi)\right)+h_{i}\left(t, \xi, v_{n}(t, \xi)+z(t, \xi)\right)$.

Arguing as in Theorem 7.7 of [19] and Proposition 6.2.2 of [10], we can assume without loss of generality that $v_{n}$ are strongly differentiable. 
By Proposition A.1 in the appendix, for $i_{t} \in\{1, \ldots, r\}, \xi_{t} \in \mathcal{O}$, such that $v_{i_{t}}\left(t, \xi_{t}\right) \operatorname{sign}\left(v_{i_{t}}\left(t, \xi_{t}\right)\right)=|v(t, \cdot)|_{E}$,

$$
\begin{aligned}
& \frac{d^{-}}{d t}\left|v_{n}(t)\right|_{E} \\
& \leq \mathcal{A}_{i_{t}} v_{n, i_{t}}\left(t, \xi_{t}\right) \operatorname{sign}\left(v_{n, i_{t}}\left(t, \xi_{t}\right)\right) \\
& \quad+g_{i_{t}, n}\left(t, \xi_{t}, v_{n, i_{t}}\left(t, \xi_{t}\right)+z_{n, i_{t}}\left(t, \xi_{t}\right)\right) \operatorname{sign}\left(v_{n, i_{t}}\left(t, \xi_{t}\right)\right) \\
& \quad+h_{i_{t}}\left(t, \xi_{t}, v_{n}\left(t, \xi_{t}\right)+z_{n}\left(t, \xi_{t}\right)\right) \operatorname{sign}\left(v_{n, i_{t}}\left(t, \xi_{t}\right)\right) .
\end{aligned}
$$

Because $\mathcal{A}_{i_{t}}$ is a second-order elliptic differential operator (see Assumption 3) and $i_{t}, \xi_{t}$ are a maximizer or minimizer, the concavity of a function at its maximum/minimum implies that

$$
\mathcal{A}_{i_{t}} v_{n, i_{t}}\left(t, \xi_{t}\right) \operatorname{sign}\left(v_{n, i_{t}}\left(t, \xi_{t}\right)\right) \leq 0 .
$$

Because $g_{i_{t}, n}\left(t, \xi_{t}, \cdot\right)$ is non-increasing and $h_{i_{t}}$ is Lipschitz continuous, by adding and subtracting $f_{i_{t}, n}\left(t, \xi_{t}, z\left(t, \xi_{t}\right)\right) \operatorname{sign}\left(v_{n, i_{t}}\left(t, \xi_{t}\right)\right)$, we see that

$$
\begin{aligned}
& \frac{d^{-}}{d t}\left|v_{n}(t)\right|_{E} \\
& \quad \leq f_{i_{t}, n}\left(t, \xi_{t}, z\left(t, \xi_{t}\right)\right) \operatorname{sign}\left(v_{n, i_{t}}\left(t, \xi_{t}\right)\right) \\
& \quad \quad+\left(g_{i_{t}, n}\left(t, \xi_{t}, v_{n, i_{t}}\left(t, \xi_{t}\right)+z_{i_{t}}\left(t, \xi_{t}\right)\right)-g_{i_{t}, n}\left(t, \xi_{t}, z_{i_{t}}\left(t, \xi_{t}\right)\right)\right) \operatorname{sign}\left(v_{n, i_{t}}\left(t, \xi_{t}\right)\right) \\
& \quad \quad+\left|h_{i_{t}}\left(t, \xi_{t}, v_{n, i_{t}}\left(t, \xi_{t}\right)+z_{i_{t}}\left(t, \xi_{t}\right)\right)-h_{i_{t}}\left(t, \xi_{t}, z\left(t, \xi_{t}\right)\right)\right| \\
& \quad \leq\left|f_{i_{t}, n}\left(t, \xi_{t}, z\left(t, \xi_{t}\right)\right)\right|+L(t)\left|v_{i_{t}}\left(t, \xi_{t}\right)\right| \\
& \quad \leq \sup _{n} \sup _{s \in[0, t]} \sup _{i \in\{1, \ldots, r\}} \sup _{\xi \in \mathcal{O}}\left|f_{i, n}(s, \xi, z(s, \xi))\right|+L(t)|v(t)|_{E} .
\end{aligned}
$$

By Grönwall's inequality and (6.7),

$$
\sup _{n} \sup _{t \in[0, T]}\left|v_{n}(t)\right|_{E} \leq C_{T} \sup _{n} \sup _{s \in[0, t]} \sup _{i \in\{1, \ldots, r\}} \sup _{\xi \in \mathcal{O}}\left|f_{i, n}(s, \xi, z(s, \xi))\right|<+\infty .
$$

Then

$$
\sup _{n}\left|u_{n}\right|_{E_{T}} \leq \sup _{n}\left(\left|v_{n}\right|_{E_{T}}+|z|_{E_{T}}\right)<+\infty .
$$

By (6.7) and (2.11)

$$
\sup _{n}\left|F_{n}\left(\cdot, u_{n}(\cdot)\right)\right|_{E_{T}} \leq\left|F\left(\cdot, u_{n}(\cdot)\right)\right|_{E_{T}}+2 L(T)\left(1+\left|u_{n}\right|_{E_{T}}\right)<+\infty
$$

By standard elliptic regularity arguments, because

$$
v_{n}(t)=\int_{0}^{t} S(t-s) F_{n}\left(s, u_{n}(s)\right) d s
$$


and $\sup _{n}\left|F_{n}\left(\cdot, u_{n}(\cdot)\right)\right|_{E_{T}}<+\infty$, there exist $\gamma>0, \beta>0$ such that

$$
\sup _{n} \sup _{i \in\{1, \ldots, r\}} \sup _{\substack{s, t \in[0, T] \\ s \neq t}} \sup _{\substack{\xi, \eta \in \mathcal{O} \\ \xi \neq \eta}} \frac{\left|v_{n, i}(t, \xi)-v_{n, i}(s, \eta)\right|}{|t-s|^{\gamma}+|x-y|^{\beta}}<+\infty .
$$

By the Arzela-Ascoli Theorem, there exists a subsequence (relabeled $v_{n}$ ) and a limit $\tilde{v} \in E_{T}$ such that $v_{n} \rightarrow \tilde{v}$ in the $E_{T}$ norm.

By the dominated convergence theorem and (6.9),

$$
\tilde{v}(t)=\int_{0}^{t} S(t-s) F(s, \tilde{v}(s)+z(s)) d s .
$$

Then $\tilde{u}:=\tilde{v}+z$ is a solution to (6.1).

Theorem 6.2 (Lipschitz continuity of $\mathcal{M}$ ). For any $T>0, \mathcal{M}$ is a Lipschitz continuous operator from $E_{T} \rightarrow E_{T}$. There exists $C_{T}>0$ depending only on $L(T)$ from Assumption 11 such that for any $z_{1}, z_{2} \in E_{T}$,

$$
\left|\mathcal{M}\left(z_{1}\right)-\mathcal{M}\left(z_{2}\right)\right|_{E_{T}} \leq C_{T}\left|z_{1}-z_{2}\right|_{E_{T}} .
$$

In particular, this theorem proves that the solution to (6.1) is unique.

Proof. Let $z_{1}, z_{2} \in E_{T}$. Let $u_{1}:=\mathcal{M}\left(z_{1}\right)$ and $u_{2}:=\mathcal{M}\left(z_{2}\right)$ be solutions to (6.1). Let $\tilde{u}=u_{1}-u_{2}$ and $\tilde{z}=z_{1}-z_{2}$. Let $v_{1}=u_{1}-z_{1}, v_{2}=u_{2}-z_{2}$. Let $\tilde{v}=v_{1}-v_{2}$. Then by the definition (6.1),

$$
\tilde{v}(t)=\int_{0}^{t} S(t-s)\left(F\left(s, v_{1}(s)+z_{1}(s)\right)-F\left(s, v_{2}(s)+z_{2}(s)\right)\right) d s .
$$

Because $\tilde{v}$ is written as a convolution with a semigroup generated by an elliptic operator, $\tilde{v}$ is weakly differentiable and

$\frac{\partial \tilde{v}_{i}}{\partial t}(t, \xi)=\mathcal{A}_{i} \tilde{v}_{i}(t, \xi)+\left(f_{i}\left(t, \xi, v_{1}(t, \xi)+z_{1}(t, \xi)\right)-f_{i}\left(t, \xi, v_{2}(t, \xi)+z_{2}(t, \xi)\right)\right)$.

By arguing as in Theorem 7.7 of [19] or Proposition 6.2.2 of [10], we can assume without loss of generality that $\tilde{v}$ is strongly differentiable.

By Proposition A.1 in the Appendix, for any $t>0$ and any index $i_{t} \in$ $\{1, \ldots, r\}$ and $\xi_{t} \in \mathcal{O}$ such that

$$
|\tilde{v}(t)|_{E}=\tilde{v}_{i_{t}}\left(t, \xi_{t}\right) \operatorname{sign}\left(\tilde{v}_{i_{t}}\left(t, \xi_{t}\right)\right),
$$




$$
\begin{aligned}
& \frac{d^{-}}{d t}|\tilde{v}(t)|_{E} \\
& \leq \mathcal{A}_{i_{t}} \tilde{v}_{i_{t}}\left(t, \xi_{t}\right) \operatorname{sign}\left(\tilde{v}_{i_{t}}\left(t, \xi_{t}\right)\right) \\
& \quad+\left(g_{i_{t}}\left(t, \xi_{t}, v_{1, i_{t}}\left(t, \xi_{t}\right)+z_{1, i_{t}}\left(t, \xi_{t}\right)\right)-g_{i_{t}}\left(t, \xi_{t}, v_{2, i_{t}}\left(t, \xi_{t}\right)+z_{2, i_{t}}\left(t, \xi_{t}\right)\right)\right) \operatorname{sign}\left(\tilde{v}_{i_{t}}\left(t, \xi_{t}\right)\right) \\
& \quad+\left(h_{i_{t}}\left(t, \xi_{t}, v_{1}\left(t, \xi_{t}\right)+z_{1}\left(t, \xi_{t}\right)\right)-h_{i_{t}}\left(t, \xi_{t}, v_{2}\left(t, \xi_{t}\right)+z_{2}\left(t, \xi_{t}\right)\right)\right) \operatorname{sign}\left(\tilde{v}_{i_{t}}\left(t, \xi_{t}\right)\right),
\end{aligned}
$$

where $g_{i}$ is the non-increasing function and $h_{i}$ is the Lipschitz continuous function from Assumption 1 .

By the ellipticity condition on $\mathcal{A}_{i_{t}}$ from Assumption 3, because $\xi_{t}$ is a maximizer or minimizer of $\tilde{v}_{i_{t}}(t, \cdot)$, the concavity of a function at its maximum/minimum implies that

$$
\mathcal{A}_{i_{t}} \tilde{v}_{i_{t}}\left(t, \xi_{t}\right) \operatorname{sign}\left(\tilde{v}_{i_{t}}\left(t, \xi_{t}\right)\right) \leq 0 .
$$

For any $t>0$ there are two cases: either

$$
\operatorname{sign}\left(\tilde{v}_{i_{t}}\left(t, \xi_{t}\right)\right)=\operatorname{sign}\left(\tilde{v}_{i_{t}}\left(t, \xi_{t}\right)+\tilde{z}_{i_{t}}\left(t, \xi_{t}\right)\right)
$$

or

$$
\operatorname{sign}\left(\tilde{v}_{i_{t}}\left(t, \xi_{t}\right)\right) \neq \operatorname{sign}\left(\tilde{v}_{i_{t}}\left(t, \xi_{t}\right)+\tilde{z}_{i_{t}}\left(t, \xi_{t}\right)\right) .
$$

If $\operatorname{sign}\left(\tilde{v}_{i_{t}}\left(t, \xi_{t}\right)\right)=\operatorname{sign}\left(\tilde{v}_{i_{t}}\left(t, \xi_{t}\right)+\tilde{z}_{i_{t}}\left(t, \xi_{t}\right)\right)$, then because $g_{i_{t}}$ is nonincreasing (2.9),

$\left(g_{i_{t}}\left(t, \xi_{t}, v_{1, i_{t}}\left(t, \xi_{t}\right)+z_{1, i_{t}}\left(t, \xi_{t}\right)\right)-g_{i_{t}}\left(t, \xi_{t}, v_{2, i_{t}}\left(t, \xi_{t}\right)+z_{2, i_{t}}\left(t, \xi_{t}\right)\right)\right) \operatorname{sign}\left(\tilde{v}_{i_{t}}\left(t, \xi_{t}\right)\right) \leq 0$.

Due to the Lipschitz continuity of $h$ (2.10),

$$
\frac{d^{-}}{d t}|\tilde{v}(t)|_{E} \leq L(t)|\tilde{v}(t)|_{E}+L(t)|\tilde{z}(t)|_{E} .
$$

On the other hand, if $\operatorname{sign}\left(\tilde{v}_{i_{t}}\left(t, \xi_{t}\right)\right) \neq \operatorname{sign}\left(\tilde{v}_{i_{t}}\left(t, \xi_{t}\right)+\tilde{z}_{i_{t}}\left(t, \xi_{t}\right)\right)$, then

$$
\left|\tilde{v}_{i_{t}}\left(t, \xi_{t}\right)\right| \leq\left|\tilde{z}_{i_{t}}\left(t, \xi_{t}\right)\right| \text {. }
$$

Because $i_{t}$ and $\xi_{t}$ maximize $\tilde{v}$, and the $E$ norm is a supremum norm, in the case where $\operatorname{sign}\left(\tilde{v}_{i_{t}}\left(t, \xi_{t}\right)\right) \neq \operatorname{sign}\left(\tilde{v}_{i_{t}}\left(t, \xi_{t}\right)+\tilde{z}_{i_{t}}\left(t, \xi_{t}\right)\right)$,

$$
|\tilde{v}(t)|_{E}=\left|\tilde{v}_{i_{t}}\left(t, \xi_{t}\right)\right| \leq\left|\tilde{z}_{i_{t}}\left(t, \xi_{t}\right)\right| \leq|\tilde{z}(t)|_{E} .
$$

We have shown that for any given $t>0$ there are only two possibilities. For any $t>0$, either

$$
\frac{d^{-}}{d t}|\tilde{v}(t)|_{E} \leq L(t)|\tilde{v}(t)|_{E}+L(t)|\tilde{z}(t)|_{E},
$$


or

$$
|\tilde{v}(t)|_{E} \leq|\tilde{z}(t)|_{E}
$$

For $t \in[0, T]$, let $\phi(t):=\max \left\{|\tilde{z}|_{E_{T}},|\tilde{v}(t)|_{E}\right\}$. Note that because $|\tilde{v}(0)|_{E}=0$ it follows that $\phi(0)=|\tilde{z}|_{E_{T}}$. Therefore,

$$
\begin{aligned}
\phi(t) & \leq|\tilde{z}|_{E_{T}}+\int_{0}^{t} \frac{d^{-}}{d s}|\tilde{v}(s)|_{E} \mathbb{1}_{\left\{|\tilde{v}(s)|_{E}>|\tilde{z}|_{E_{T}}\right\}} d s \\
& \leq C_{T}|\tilde{z}|_{E_{T}}+L(T) \int_{0}^{t}\left(|\tilde{v}(s)|_{E}+|\tilde{z}(s)|_{E}\right) d s \\
& \leq C_{T}|\tilde{z}|_{E_{T}}+2 L(T) \int_{0}^{t} \phi(s) d s .
\end{aligned}
$$

By Grönwall's inequality, there exists $C_{T}>0$ such that

$$
\sup _{t \in[0, T]} \phi(t) \leq C_{T}|\tilde{z}|_{E_{T}}
$$

Therefore

$$
|\tilde{v}|_{E_{T}} \leq C_{T}|\tilde{z}|_{E_{T}}
$$

Because $\tilde{u}(t)=\tilde{v}(t)+\tilde{z}(t)$,

$$
|\tilde{u}|_{E_{T}} \leq\left(C_{T}+1\right)|\tilde{z}|_{E_{T}}
$$

proving our result.

\section{Existence and uniqueness of the solution to con- trolled stochastic reaction diffusion equations}

In this section we prove that under Assumptions 1, 2, 3, and 4, the solutions to the controlled SPDE (2.30) exist and are unique. Because our assumptions are weaker than previous results, these existence and uniqueness results cannot be found in the literature. The existence of the mild solutions to the uncontrolled SPDE (2.29) is a corollary obtained by using the trivial control $u \equiv 0$. Notice that our assumptions are strictly weaker than those in [11] or [13].

Theorem 7.1. For any $x \in E, N>0, u \in \mathscr{A}_{N}$, and $\varepsilon>0$, there exists a unique solution $X_{x}^{\varepsilon, u}$ to (2.30) and the solution is $E_{T}$ valued. 
Proof. We build a contraction mapping. Let $\hat{E}_{T}$ denote the collection of continuous random fields $\psi: \Omega \times[0, T] \times \overline{\mathcal{O}} \times\{1, \ldots, r\} \rightarrow \mathbb{R}$ that are adapted to the filtration $\mathcal{F}_{t}$.

By the definition of $\mathcal{M}(6.1), X_{x}^{\varepsilon, u}$ is a solution to (2.30) if and only if it satisfies

$$
X_{x}^{\varepsilon, u}=\mathcal{M}\left(S(\cdot) x+Y^{u}\left(X_{x}^{\varepsilon, u}\right)+\sqrt{\varepsilon} Z\left(X_{x}^{\varepsilon, u}\right)\right)
$$

where for any $\psi \in \hat{E}_{T}, Y^{u}(\psi) \in \hat{E}_{T}$ and $Z(\psi) \in \hat{E}_{T}$ are defined by

$$
Y^{u}(\psi)(t)=\int_{0}^{t} S(t-s) R(s, \psi(s)) Q u(s) d s
$$

and

$$
Z(\psi)(t)=\int_{0}^{t} S(t-s) R(s, \psi(s)) d w(s) .
$$

Let $\mathcal{K}_{x}^{\varepsilon, u}: \hat{E}_{T} \rightarrow \hat{E}_{T}$ be defined by

$$
\mathcal{K}_{x}^{\varepsilon, u}(\psi)=\mathcal{M}\left(S(\cdot) x+Y^{u}(\psi)+\sqrt{\varepsilon} Z(\psi)\right) .
$$

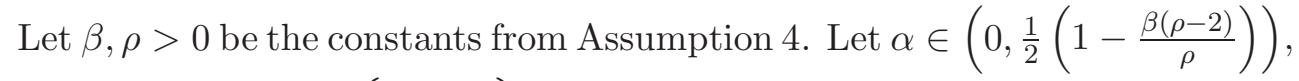
$\gamma \in(0, \alpha)$, and $p>\max \left\{\frac{1}{\alpha-\gamma}, \frac{d}{\gamma}\right\}$. By Theorem 6.2, there exists a constant $C_{T}>0$ such that for $\psi_{1}, \psi_{2} \in L^{p}\left(\Omega: E_{T}\right)$,

$$
\begin{aligned}
& \mathbb{E}\left|\mathcal{K}_{x}^{\varepsilon, u}\left(\psi_{1}\right)-\mathcal{K}_{x}^{\varepsilon, u}\left(\psi_{2}\right)\right|_{E_{T}}^{p} \\
& \leq C_{T}\left(\mathbb{E}\left|Y^{u}\left(\psi_{1}\right)-Y^{u}\left(\psi_{2}\right)\right|_{E_{T}}^{p}+\varepsilon^{\frac{p}{2}} \mathbb{E}\left|Z\left(\psi_{1}\right)-Z\left(\psi_{2}\right)\right|_{E_{T}}^{p}\right) .
\end{aligned}
$$

By Theorem B.5 in the Appendix,

$$
\begin{aligned}
& \mathbb{E}\left|Z\left(\psi_{1}\right)-Z\left(\psi_{2}\right)\right|_{E_{T}}^{p} \\
& \leq C \mathbb{E} \int_{0}^{T}\left(\int_{0}^{t}(t-s)^{-2 \alpha-\frac{\beta(\rho-2)}{\rho}} \max _{i \in\{1, \ldots, r\}}\left|R_{i} \cdot\left(s, \psi_{1}(s)\right)-R_{i \cdot}\left(s, \psi_{2}(s)\right)\right|_{E}^{2} d s\right)^{\frac{p}{2}} d t .
\end{aligned}
$$

By the Lipschitz continuity of $R$, and the fact that $-2 \alpha-\frac{\beta(\rho-2)}{\rho}>-1$,

$$
\mathbb{E}\left|Z\left(\psi_{1}\right)-Z\left(\psi_{2}\right)\right|_{E_{T}}^{p} \leq C_{T} \int_{0}^{T} \mathbb{E}\left|\psi_{1}-\psi_{2}\right|_{E_{t}}^{p} d t
$$

Similarly, by Theorem B.6, because $u \in \mathscr{A}_{N}$,

$$
\mathbb{E}\left|Y^{u}\left(\psi_{1}\right)-Y^{u}\left(\psi_{2}\right)\right|_{E_{T}}^{p} \leq C_{T} N^{\frac{p}{2}} \int_{0}^{T} \mathbb{E}\left|\psi_{1}-\psi_{2}\right|_{E_{t}}^{p} d t .
$$


By (7.4), (7.5), and (7.6),

$$
\mathbb{E}\left|\mathcal{K}_{x}^{\varepsilon, u}\left(\psi_{1}\right)-\mathcal{K}_{x}^{\varepsilon, u}\left(\psi_{2}\right)\right|_{E_{T}}^{p} \leq C_{T}\left(\varepsilon^{\frac{p}{2}}+N^{\frac{p}{2}}\right) \int_{0}^{T}\left|\psi_{1}-\psi_{2}\right|_{E_{t}}^{p} d t .
$$

There exists a $T_{0}$ small enough so that $C_{T_{0}} T_{0}\left(\varepsilon^{\frac{p}{2}}+N^{\frac{p}{2}}\right)<1$. Then $\mathcal{K}_{x}^{\varepsilon, u}$ is a contraction mapping on $L^{p}\left(\Omega: E_{T_{0}}\right)$ and there exists a unique fixed point $X_{x}^{\varepsilon, u}$ solving (7.1) for $t \in\left[0, T_{0}\right]$. This argument can be repeated on $\left[T_{0}, 2 T_{0}\right],\left[2 T_{0}, 3 T_{0}\right]$ and so forth to prove that there exists a unique global solution to the control equation (7.1).

Corollary 7.2. For any $x \in E$ and $\varepsilon>0$, there exists a unique global mild solution to the uncontrolled SPDE $X_{x}^{\varepsilon}(2.29)$.

Proof. This is immediate by using the trivial control $u \equiv 0$ in Theorem 7.1 because $X_{x}^{\varepsilon}=X_{x}^{\varepsilon, 0}$.

Next we prove that the solutions to the control equation (7.1) are bounded in $L^{p}\left(\Omega: E_{T}\right)$ uniformly for $u \in \mathscr{A}_{N}$, and bounded subsets of $\varepsilon>0$ and bounded subsets of $x \in E$.

Theorem 7.3. For $T>0$ and $p>1$, there exists $C_{T, p}>0$ such that for any $N>0, u \in \mathscr{A}_{N}, \varepsilon>0$, and $x \in E$,

$$
\mathbb{E}\left|X_{x}^{\varepsilon, u}\right|_{E_{T}}^{p} \leq C_{T, p} e^{C_{T, p}\left(\varepsilon^{\frac{p}{2}}+N^{\frac{p}{2}}\right)}\left(1+|x|_{E}^{p}\right) .
$$

Proof. By (7.1) and the Lipschitz continuity of $\mathcal{M}$, Theorem 6.2,

$$
\begin{aligned}
& \mathbb{E}\left|X_{x}^{\varepsilon, u}\right|_{E_{T}}^{p}=\mathbb{E}\left|\mathcal{M}\left(S(\cdot) x+Y^{u}\left(X_{x}^{\varepsilon, u}\right)+\sqrt{\varepsilon} Z\left(X_{x}^{\varepsilon, u}\right)\right)\right|_{E_{T}}^{p} \\
& \leq C_{p} \mathbb{E}\left|\mathcal{M}\left(S(\cdot) x+Y^{u}\left(X_{x}^{\varepsilon, u}\right)+\sqrt{\varepsilon} Z\left(X_{x}^{\varepsilon, u}\right)\right)-\mathcal{M}(0)\right|_{E_{T}}^{p}+C_{p}|\mathcal{M}(0)|_{E_{T}}^{p} \\
& \leq C_{T, p}\left(1+|x|_{E}^{p}+\mathbb{E}\left|Y^{u}\left(X_{x}^{\varepsilon, u}\right)\right|_{E_{T}}^{p}+\varepsilon^{\frac{p}{2}} \mathbb{E}\left|Z\left(X_{x}^{\varepsilon, u}\right)\right|_{E_{T}}^{p}\right) .
\end{aligned}
$$

By Theorem B.5, Theorem B.6 and the fact that $R$ has linear growth, for large enough $p$,

$$
\mathbb{E}\left|X_{x}^{\varepsilon, u}\right|_{E_{T}}^{p} \leq C_{T, p}\left(1+|x|_{E}+\left(\varepsilon^{\frac{p}{2}}+N^{\frac{p}{2}}\right) \int_{0}^{T} \mathbb{E}\left|X_{x}^{\varepsilon, u}\right|_{E_{t}}^{p} d t\right) .
$$

The result follows by Grönwall's inequality.

Corollary 7.4. For $T>0$ and $p>1$, there exists $C_{T, p}>0$ such that for any $\varepsilon>0$, and $x \in E$,

$$
\mathbb{E}\left|X_{x}^{\varepsilon}\right|_{E_{T}}^{p} \leq C_{T, p} e^{C_{T, p} \varepsilon^{\frac{p}{2}}}\left(1+|x|_{E}^{p}\right) .
$$


Proof. This is an immediate consequence of Theorem 7.3 and the fact that $X_{x}^{\varepsilon}=X_{x}^{\varepsilon, 0}$.

\section{Uniform large deviations principle over bounded subsets of $E-$ Proof of Theorem 4.1}

In this section, we use Corollary 3.3 to prove that the mild solutions $\left\{X_{x}^{\varepsilon}\right\}_{\substack{\varepsilon>0 \\ x \in E}}$ to (1.1) satisfy a uniform large deviations principle that is uniform over bounded subsets of $E$.

Proof of Theorem 4.1. By Corollary 3.3, it is sufficient to prove that for any $K>0, N>0$, and $\delta>0$,

$$
\lim _{\varepsilon \rightarrow 0} \sup _{|x|_{E} \leq K} \sup _{u \in \mathscr{A}_{N}} \mathbb{P}\left(\left|X_{x}^{\varepsilon, u}-X_{x}^{0, u}\right|_{E_{T}}>\delta\right)=0 .
$$

Let

$$
Y_{x}^{\varepsilon, u}(t)=\int_{0}^{t} S(t-s) R\left(s, X_{x}^{\varepsilon, u}(s)\right) Q u(s) d s
$$

and

$$
Z_{x}^{\varepsilon, u}(t)=\int_{0}^{t} S(t-s) R\left(s, X_{x}^{\varepsilon, u}(s)\right) d w(s) .
$$

Using this notation,

$$
X_{x}^{\varepsilon, u}=\mathcal{M}\left(S(\cdot) x+Y_{x}^{\varepsilon, u}+\sqrt{\varepsilon} Z_{x}^{\varepsilon, u}\right),
$$

where $\mathcal{M}: E_{T} \rightarrow E_{T}$ solves (6.1).

By the Lipschitz continuity of $\mathcal{M}$ (Theorem 6.2),

$$
\left|X_{x}^{\varepsilon, u}-X_{x}^{0, u}\right|_{E_{T}} \leq C_{T}\left|Y_{x}^{\varepsilon, u}-Y_{x}^{0, u}\right|_{E_{T}}+C_{T} \sqrt{\varepsilon}\left|Z_{x}^{\varepsilon, u}\right|_{E_{T}} .
$$

By Theorem B.6. for $u \in \mathcal{A}_{N}, \varepsilon>0$, and $x \in E$,

$$
\begin{aligned}
& \left|Y_{x}^{\varepsilon, u}-Y_{x}^{0, u}\right|_{E_{T}} \\
& \leq C N^{\frac{1}{2}} \sup _{t \in[0, T]}\left(\int_{0}^{t}(t-s)^{-\frac{\beta(\rho-2)}{\rho}} \max _{i \in\{1, \ldots, r\}}\left|R_{i \cdot}\left(s, X_{x}^{\varepsilon, u}(s)\right)-R_{i \cdot}\left(s, X_{x}^{0, u}(s)\right)\right|_{E}^{2} d s\right)^{\frac{1}{2}} .
\end{aligned}
$$

By the Lipschitz continuity of $R$ (Assumption 2),

$$
\begin{aligned}
& \left|Y_{x}^{\varepsilon, u}-Y_{x}^{0, u}\right|_{E_{T}} \\
& \leq C N^{\frac{1}{2}} \sup _{t \in[0, T]}\left(\int_{0}^{t}(t-s)^{-\frac{\beta(\rho-2)}{\rho}}\left|X_{x}^{\varepsilon, u}(s)-X_{x}^{0, u}(s)\right|_{E}^{2} d s\right)^{\frac{1}{2}} .
\end{aligned}
$$


By Assumption (2.20), $\frac{\beta(\rho-2)}{\rho}<1$. For $p>\frac{2}{1-\frac{\beta(\rho-2)}{\rho}}$, the Hölder inequality shows that

$$
\left|Y_{x}^{\varepsilon, u}-Y_{x}^{0, u}\right|_{E_{T}}^{p} \leq C_{p, T} N^{\frac{p}{2}} \int_{0}^{T}\left|X_{x}^{\varepsilon, u}-X_{x}^{0, u}\right|_{E_{t}}^{p} d t
$$

Let $\alpha \in\left(0, \frac{1}{2}\left(1-\frac{\beta(\rho-2)}{\rho}\right)\right), \gamma \in(0, \alpha)$, and $p>\max \left\{\frac{1}{\alpha-\gamma}, \frac{d}{\gamma}\right\}$. By Theorem B.5.

$$
\begin{aligned}
& \mathbb{E}\left|Z_{x}^{\varepsilon, u}\right|_{E_{T}}^{p} \\
& \leq C_{T, p} \mathbb{E} \int_{0}^{T}\left(\int_{0}^{t}(t-s)^{-2 \alpha-\frac{\beta(\rho-2)}{\rho}} \max _{i \in\{1, \ldots r\}}\left|R_{i \cdot}\left(s, X_{x}^{\varepsilon, u}(s)\right)\right|_{E}^{2} d s\right)^{\frac{p}{2}} d t .
\end{aligned}
$$

By the linear growth of $R$, and the fact that $-2 \alpha-\frac{\beta(\rho-2)}{\rho}>-1$,

$$
\mathbb{E}\left|Z_{x}^{\varepsilon, u}\right|_{E_{T}}^{p} \leq C_{T, p}\left(1+\mathbb{E}\left|X_{x}^{\varepsilon, u}\right|_{E_{T}}^{p}\right)
$$

By (7.7),

$$
\mathbb{E}\left|Z_{x}^{\varepsilon, u}\right|_{E_{T}}^{p} \leq C_{T, p} e^{\left(\varepsilon^{\frac{p}{2}}+N^{\frac{p}{2}}\right) C_{T, p}}\left(1+|x|_{E}^{p}\right) .
$$

Therefore by (8.4), (8.5), and (8.7),

$$
\begin{aligned}
& \mathbb{E}\left|X_{x}^{\varepsilon, u}-X_{x}^{0, u}\right|_{E_{T}}^{p} \\
& \leq C_{T, p} N^{\frac{p}{2}} \int_{0}^{T} \mathbb{E}\left|X_{x}^{\varepsilon, u}-X_{x}^{0, u}\right|_{E_{t}}^{p} d t+C_{T, p} \varepsilon^{\frac{p}{2}} e^{\left(\varepsilon^{\frac{p}{2}}+N^{\frac{p}{2}}\right) C_{T, p}}\left(1+|x|_{E}^{p}\right) .
\end{aligned}
$$

By Grönwall's inequality, for any $K>0$,

$$
\sup _{|x|_{E} \leq K} \sup _{u \in \mathcal{A}_{N}} \mathbb{E}\left|X_{x}^{\varepsilon, u}-X_{x}^{0, u}\right|_{E_{T}}^{p} \leq \varepsilon^{\frac{p}{2}} C_{T, p} e^{\left(\varepsilon^{\frac{p}{2}}+N^{\frac{p}{2}}\right) C_{T, p}}\left(1+K^{p}\right) .
$$

By the Chebyshev inequality,

$$
\lim _{\varepsilon \rightarrow 0} \sup _{|x|_{E} \leq K} \sup _{u \in \mathcal{A}_{N}} \mathbb{P}\left(\left|X_{x}^{\varepsilon, u}-X_{x}^{0, u}\right|_{E_{T}}>\delta\right)=0 .
$$

Then Theorem 4.1 is a consequence of Corollary 3.3 . 


\section{Uniform large deviations when $\sigma$ is uniformly bounded - Proof of Theorem 4.2}

Proof of Theorem 4.2. By Corollary 3.3 it suffices to show that for any $\delta>0$ and $N>0$,

$$
\lim _{\varepsilon \rightarrow 0} \sup _{x \in E} \sup _{u \in \mathcal{A}_{N}} \mathbb{P}\left(\left|X_{x}^{\varepsilon, u}-X_{x}^{0, u}\right|_{E_{T}}>\delta\right)=0 .
$$

Let $Y_{x}^{\varepsilon, u}$ and $Z_{x}^{\varepsilon, u}$ be the solutions to (8.2) and (8.3). Then

$$
X_{x}^{\varepsilon, u}=\mathcal{M}\left(S(\cdot) x+Y_{x}^{\varepsilon, u}+\sqrt{\varepsilon} Z_{x}^{\varepsilon, u}\right) .
$$

By the Lipschitz continuity of $\mathcal{M}$ (Theorem 6.2), (8.4) holds. By Theorem B.6. (8.5) holds. By Theorem B.5, for large enough $p>\frac{2}{1-\frac{\beta(\rho-2)}{\rho}}$, there exists $C_{T, p}>0$ such that

$$
\begin{aligned}
& \mathbb{E}\left|Z_{x}^{\varepsilon, u}\right|_{E_{T}}^{p} \\
& \leq C_{T, p} \mathbb{E} \int_{0}^{T}\left(\int_{0}^{t}(t-s)^{-2 \alpha-\frac{\beta(\rho-2)}{\rho}} \max _{i \in\{1, \ldots . r\}}\left|R_{i \cdot}\left(s, X_{x}^{\varepsilon, u}(s)\right)\right|_{E}^{2} d s\right)^{\frac{p}{2}} d t .
\end{aligned}
$$

By (4.5),

$$
\sup _{s \in[0, T]} \sup _{x \in E} \sup _{n \in\{1, \ldots r\}}\left|R_{\cdot n}(s, X)\right|_{E} \leq L(T) .
$$

Because $-2 \alpha-\frac{\beta(\rho-2)}{\rho}>-1$,

$$
\sup _{\varepsilon \in(0,1)} \sup _{x \in E} \sup _{u \in \mathcal{A}_{N}} \mathbb{E}\left|Z_{x}^{\varepsilon, u}\right|_{E_{T}}^{p} \leq C_{T, p}
$$

By (8.4), (8.5), and (9.2), there exists $C_{T, p}>0$ such that for any $x \in E$, $\varepsilon>0, N>0$, and $u \in \mathscr{A}_{N}$,

$$
\mathbb{E}\left|X_{x}^{\varepsilon, u}-X_{x}^{0, u}\right|_{E_{T}}^{p} \leq C_{T, p} N^{\frac{p}{2}} \int_{0}^{T} \mathbb{E}\left|X_{x}^{\varepsilon, u}-X_{x}^{0, u}\right|_{E_{t}}^{p} d t+C_{T, p} \varepsilon^{\frac{p}{2}} .
$$

By Grönwall's inequality,

$$
\mathbb{E}\left|X_{x}^{\varepsilon, u}-X_{x}^{0, u}\right|_{E_{T}}^{p} \leq C_{T, p} \varepsilon^{\frac{p}{2}} e^{C_{p, T} N^{\frac{p}{2}} T} .
$$

This estimate is uniform with respect to $x \in E$ and therefore

$$
\lim _{\varepsilon \rightarrow 0} \sup _{x \in E} \sup _{u \in \mathscr{A}_{N}} \mathbb{E}\left|X_{x}^{\varepsilon, u}-X_{x}^{0, u}\right|_{E_{T}}^{p}=0 .
$$

By the Chebyshev inequality,

$$
\lim _{\varepsilon \rightarrow 0} \sup _{x \in E} \sup _{u \in \mathcal{A}_{N}} \mathbb{P}\left(\left|X_{x}^{\varepsilon, u}-X_{x}^{0, u}\right|_{E_{T}}>\delta\right)=0 .
$$

Then Theorem 4.2 is a consequence of Corollary 3.3 . 


\section{Uniform large deviations when $f$ has super- linear dissipativity - Proof of Theorem 4.3}

Proof of Theorem 4.3. By Corollary 3.3, it is sufficient to show that for any $\delta>0$ and $N>0$

$$
\lim _{\varepsilon \rightarrow 0} \sup _{x \in E} \sup _{u \in \mathscr{A}_{N}} \mathbb{P}\left(\left|X_{x}^{\varepsilon, u}-X_{x}^{0, u}\right|_{E_{T}}>\delta\right)=0 .
$$

For $x \in E$, define $\mathcal{M}_{x}: E_{T} \rightarrow E_{T}$ by

$$
\mathcal{M}_{x}(\varphi):=\mathcal{M}(S(\cdot) x+\varphi) .
$$

Under the super-linear dissipativity assumption (Assumption 6 ), $\mathcal{M}_{x}$ satisfies certain bounds that are independent of the initial condition $x$. These results are presented in Appendix C.

We observe that $X_{x}^{\varepsilon, u}$ can be written as

$$
X_{x}^{\varepsilon, u}=\mathcal{M}_{x}\left(Y_{x}^{\varepsilon, u}+\sqrt{\varepsilon} Z_{x}^{\varepsilon, u}\right)
$$

where

$$
\begin{aligned}
Y_{x}^{\varepsilon, u}(t) & =\int_{0}^{t} S(t-s) R\left(s, X_{x}^{\varepsilon, u}(s)\right) Q u(s) d s \\
Z_{x}^{\varepsilon, u}(t) & =\int_{0}^{t} S(t-s) R\left(s, X_{x}^{\varepsilon, u}(s)\right) d w(s)
\end{aligned}
$$

By Theorem B.5, for $u \in \mathscr{A}_{N}, \varepsilon>0, x \in E$, and any $\alpha \in\left(0, \frac{1}{2}\left(1-\frac{\beta(\rho-2)}{\rho}\right)\right)$, $\gamma \in(0, \alpha)$, and $p>\max \left\{\frac{1}{\alpha-\gamma}, \frac{d}{\gamma}\right\}$,

$$
\begin{aligned}
& \mathbb{E}\left|Z_{x}^{\varepsilon, u}\right|_{E_{T}}^{p} \\
& \leq C_{T, p} \mathbb{E} \int_{0}^{T}\left(\int_{0}^{t}(t-s)^{-2 \alpha-\frac{\beta(\rho-2)}{\rho}} \max _{i \in\{1, \ldots, r\}}\left|R_{i \cdot}\left(s, X_{x}^{\varepsilon, u}(s)\right)\right|_{E}^{2} d s\right)^{\frac{p}{2}} d t .
\end{aligned}
$$

By the assumed growth rate on $\sigma$ (and therefore $R$ ) in Assumption 6 ,

$$
\begin{aligned}
& \mathbb{E}\left|Z_{x}^{\varepsilon, u}\right|_{E_{T}}^{p} \\
& \leq C_{T, p} \int_{0}^{T} \mathbb{E}\left(\int_{0}^{t}(t-s)^{-2 \alpha-\frac{\beta(\rho-2)}{\rho}}\left(1+\left|X_{x}^{\varepsilon, u}(s)\right|_{E}^{2 \nu}\right) d s\right)^{\frac{p}{2}} d t .
\end{aligned}
$$

By the fact that $X_{x}^{\varepsilon}=\mathcal{M}_{x}\left(Y_{x}^{\varepsilon}+Z_{x}^{\varepsilon}\right)$ and (‥2),

$$
\left|X_{x}^{\varepsilon}(t)\right|_{E} \leq C_{t}\left(1+t^{-\frac{1}{m-1}}+\left|Y_{x}^{\varepsilon, u}+\sqrt{\varepsilon} Z_{x}^{\varepsilon, u}\right|_{E_{t}}\right)
$$


Therefore,

$$
\begin{aligned}
& \mathbb{E}\left|Z_{x}^{\varepsilon, u}\right|_{E_{T}}^{p} \\
& \leq C_{T, p} \int_{0}^{T} \mathbb{E}\left(\int_{0}^{t}(t-s)^{-2 \alpha-\frac{\beta(\rho-2)}{\rho}}\left(1+s^{-\frac{2 \nu}{m-1}}+\left|Y_{x}^{\varepsilon, u}+\sqrt{\varepsilon} Z_{x}^{\varepsilon, u}\right|_{E_{s}}^{2 \nu}\right) d s\right)^{\frac{p}{2}} d t .
\end{aligned}
$$

By (4.10) we can choose

$$
\alpha:=\frac{1}{2}\left(1-\frac{\beta(\rho-2)}{\rho}-\frac{2 \nu}{m-1}\right) \in\left(0, \frac{1}{2}\right) .
$$

Then for any $t>0$, by the properties of the Beta function, for any $t>0$,

$$
\begin{aligned}
& \int_{0}^{t}(t-s)^{-2 \alpha-\frac{\beta(\rho-2)}{\rho}} s^{-\frac{2 \nu}{m-1}} d s \\
& =\int_{0}^{1}(1-s)^{-2 \alpha-\frac{\beta(\rho-2)}{\rho}} s^{-\frac{2 \nu}{m-1}} d s \\
& =\frac{\pi}{\sin \left(\frac{2 \nu \pi}{m-1}\right)} .
\end{aligned}
$$

Furthermore, by Assumption [6, $\nu \in[0,1]$. There will exist large enough constants such that

$$
\mathbb{E}\left|Z_{x}^{\varepsilon, u}\right|_{E_{T}}^{p} \leq C_{T, p}\left(1+\int_{0}^{T} \mathbb{E}\left|Y_{x}^{\varepsilon, u}+\sqrt{\varepsilon} Z_{x}^{\varepsilon, u}\right|_{E_{t}}^{p} d t\right) .
$$

Similarly, by Theorem B.6 and (C.2)

$$
\begin{aligned}
\left|Y_{x}^{\varepsilon, u}\right|_{E_{T}} \leq & C_{T} N^{\frac{1}{2}} \sup _{t \in[0, T]} \int_{0}^{t}(t-s)^{-\frac{\beta(\rho-2)}{\rho}}\left(1+s^{-\frac{2 \nu}{m-1}}+\left|Y_{x}^{\varepsilon, u}+\sqrt{\varepsilon} Z_{x}^{\varepsilon, u}\right|_{E_{s}}^{2}\right) d s \\
& \leq C_{T} N^{\frac{1}{2}}\left(1+\sup _{t \in[0, T]} \int_{0}^{t}(t-s)^{-\frac{\beta(\rho-2)}{\rho}}\left(\left|Y_{x}^{\varepsilon, u}+\sqrt{\varepsilon} Z_{x}^{\varepsilon, u}\right|_{E_{s}}^{2}\right) d s\right) .
\end{aligned}
$$

By a Hölder inequality,

$$
\left|Y_{x}^{\varepsilon, u}\right|_{E_{T}}^{p} \leq C_{T, p} N^{\frac{p}{2}}\left(1+\int_{0}^{T}\left|Y_{x}^{\varepsilon, u}+\sqrt{\varepsilon} Z_{x}^{\varepsilon, u}\right|_{E_{t}}^{p} d t\right) .
$$

Combining (10.7) and (10.8),

$$
\mathbb{E}\left|Y_{x}^{\varepsilon, u}+\sqrt{\varepsilon} Z_{x}^{\varepsilon, u}\right|_{E_{T}}^{p} \leq C_{T, p}\left(N^{\frac{p}{2}}+\varepsilon^{\frac{p}{2}}\right)\left(1+\int_{0}^{T}\left|Y_{x}^{\varepsilon, u}+\sqrt{\varepsilon} Z_{x}^{\varepsilon, u}\right|_{E_{t}}^{p} d t\right) .
$$


By Grönwall's inequality,

$$
\mathbb{E}\left|Y_{x}^{\varepsilon, u}+\sqrt{\varepsilon} Z_{x}^{\varepsilon, u}\right|_{E_{T}}^{p} \leq C_{T, p}\left(N^{\frac{p}{2}}+\varepsilon^{\frac{p}{2}}\right) e^{C_{T, p}\left(N^{\frac{p}{2}}+\varepsilon^{\frac{p}{2}}\right)} .
$$

From Theorem B.5, (10.6), and (10.9) we can conclude that

$$
\begin{aligned}
& \varepsilon^{\frac{p}{2}} \mathbb{E}\left|Z_{x}^{\varepsilon, u}\right|_{E_{T}}^{p} \\
& \leq C_{T, p} \varepsilon^{\frac{p}{2}} \int_{0}^{T} \mathbb{E}\left(\int_{0}^{t}(t-s)^{-2 \alpha-\frac{\beta(\rho-2)}{\rho}}\left(1+s^{-\frac{2 \nu}{m-1}}+\left|Y_{x}^{\varepsilon, u}+\sqrt{\varepsilon} Z_{x}^{\varepsilon, u}\right|_{E_{s}}^{2 \nu}\right) d s\right)^{\frac{p}{2}} d t \\
& \leq \varepsilon^{\frac{p}{2}} C_{T, p}\left(1+N^{\frac{p}{2}}+\varepsilon^{\frac{p}{2}}\right) e^{C_{T, p}\left(N^{\frac{p}{2}}+\varepsilon^{\frac{p}{2}}\right)} .
\end{aligned}
$$

The above bound is uniform over $x \in E$.

The remainder of the proof is very similar to the proofs of Theorems 4.1 and 4.2. By the Lipschitz continuity of $\mathcal{M}$ (Theorem 6.2),

$$
\left|X_{x}^{\varepsilon, u}-X_{x}^{0, u}\right|_{E_{T}} \leq C_{T}\left|Y_{x}^{\varepsilon, u}-Y_{x}^{0, u}\right|_{E_{T}}+C_{T} \sqrt{\varepsilon}\left|Z_{x}^{\varepsilon, u}\right|_{E_{T}}
$$

By (8.5),

$$
\left|Y_{x}^{\varepsilon, u}-Y_{x}^{0, u}\right|_{E_{T}}^{p} \leq C_{T, p} N^{\frac{p}{2}} \int_{0}^{T}\left|X_{x}^{\varepsilon, u}-X_{x}^{0, u}\right|_{E_{t}}^{p} d t
$$

Therefore, (10.10) implies

$$
\begin{aligned}
& \mathbb{E}\left|X_{x}^{\varepsilon, u}-X_{x}^{0, u}\right|_{E_{T}}^{p} \\
& \leq C_{T, p} N^{\frac{p}{2}} \int_{0}^{T} \mathbb{E}\left|X_{x}^{\varepsilon, u}-X_{x}^{0, u}\right|_{E_{t}}^{p} d t+\varepsilon^{\frac{p}{2}} C_{T, p}\left(N^{\frac{p}{2}}+\varepsilon^{\frac{p}{2}}\right) e^{C_{T, p}\left(N^{\frac{p}{2}}+\varepsilon^{\frac{p}{2}}\right)} .
\end{aligned}
$$

By Grönwall's inequality, there exists $C_{N, T, p}>0$ such that for all $\varepsilon \in(0,1)$,

$$
\sup _{x \in E} \sup _{u \in \mathcal{A}_{N}} \mathbb{E}\left|X_{x}^{\varepsilon, u}-X_{x}^{0, u}\right|_{E_{T}}^{p} \leq C_{N, T, p} \varepsilon^{\frac{p}{2}}
$$

By the Chebyshev inequality,

$$
\lim _{\varepsilon \rightarrow 0} \sup _{x \in E} \sup _{u \in \mathcal{A}_{N}} \mathbb{P}\left(\left|X_{x}^{\varepsilon, u}-X_{x}^{0, u}\right|_{E_{T}}>\delta\right)=0 .
$$

Then Theorem 4.3 is a consequence of Corollary 3.3 . 


\section{A The left derivative of the supremum norm}

Let $E=\{x \in C(\overline{\mathcal{O}} \times\{1, \ldots, r\}): x(\xi)=0$ for $\xi \in \partial \mathcal{O}\}$ endowed with the supremum norm

$$
|x|_{E}:=\sup _{\xi \in \overline{\mathcal{O}}} \sup _{i \in\{1, \ldots, r\}}\left|x_{i}(\xi)\right| .
$$

For a real-valued function $\phi:[0, T] \rightarrow \mathbb{R}$, define the left derivative by

$$
\frac{d^{-} \phi}{d t}(t)=\limsup _{h \downarrow 0} \frac{\phi(t)-\phi(t-h)}{h} .
$$

Let $\psi:[0, T] \times \overline{\mathcal{O}} \times\{1, \ldots r\} \rightarrow \mathbb{R}$ be differentiable in its first argument.

Proposition A.1 (Proposition D.4 of [19]). Assume that $\psi:[0, T] \times \overline{\mathcal{O}} \times$ $\{1, \ldots r\}$ has a continuous partial derivative in time. The left-derivative of the $E$ norm is bounded above by

$$
\frac{d^{-}}{d t}|\psi \cdot(t, \cdot)|_{E} \leq\left[\frac{\partial \psi}{\partial t}\right]_{i_{t}}\left(t, \xi_{t}\right) \operatorname{sign}\left(\psi_{i_{t}}\left(t, \xi_{t}\right)\right)
$$

for any maximizer/minimizer $\left(i_{t}, \xi_{t}\right) \in\{1, \ldots, r\} \times \overline{\mathcal{O}}$ such that

$$
\left|\psi_{i_{t}}\left(t, \xi_{t}\right)\right|=|\psi \cdot(t, \cdot)|_{E} .
$$

Proof. Fix $t>0$. Let $\left(i_{t}, \xi_{t}\right) \in\{1, \ldots . r\} \times \overline{\mathcal{O}}$ be a maximizer $/$ minimizer of $\psi \cdot(t, \cdot)$ such that

$$
\left|\psi_{i_{t}}\left(t, \xi_{t}\right)\right|=|\psi|_{E}
$$

Notice that for another time $h \in(0, t)$,

$$
\psi_{i_{t}}\left(t-h, \xi_{t}\right) \operatorname{sign}\left(\psi_{i_{t}}\left(t, \xi_{t}\right)\right) \leq|\psi \cdot(t-h, \cdot)|_{E} .
$$

The left-derivative of $|\psi(t)|_{E}$ is

$$
\begin{aligned}
& \frac{d^{-}}{d t}|\psi(t)|_{E} \\
& =\limsup _{h \downarrow 0} \frac{|\psi(t)|_{E}-|\psi(t+h)|_{E}}{h} \\
& \leq \limsup _{h \downarrow 0} \frac{\left(\psi_{i_{t}}\left(t, \xi_{t}\right)-\psi_{i_{t}}\left(t-h, \xi_{t}\right)\right) \operatorname{sign}\left(\psi_{i_{t}}\left(t, \xi_{t}\right)\right)}{h} \\
& =\left[\frac{\partial \psi}{\partial t}\right]_{i_{t}}\left(t, \xi_{t}\right) \operatorname{sign}\left(\psi_{i_{t}}\left(t, \xi_{t}\right)\right) .
\end{aligned}
$$




\section{B Continuity in time and space of the stochastic convolution}

This appendix collects some results from [11,12] about the continuity of stochastic convolution terms.

Assume Assumptions 3, and 4. Let $E_{T}$ be defined by (2.4). For arbitrary $\sigma \in E_{T}$, define the multiplication operators $R_{n}:[0, T] \rightarrow \mathscr{L}\left(L^{2}(\mathcal{O})\right)$ such that for any $n \in\{1, \ldots r\}, t \in[0, T]$, and $\xi \in \mathcal{O}$, and $f \in L^{2}(\mathcal{O})$.

$$
\left[R_{n}(t) f\right](\xi):=\sigma_{n}(t, \xi) f(\xi) .
$$

In this appendix, we investigate the continuity in time and space of the stochastic convolutions

$$
Z_{i}(t):=\int_{0}^{t} S_{i}(t-s) \sum_{n=1}^{r} R_{n}(s) d w_{n}(s)
$$

solving

$$
d Z_{i}(t)=A_{i} Z_{i}(t)+\sum_{n=1}^{r} R_{n}(t) d w_{n}(t) .
$$

In these expressions, $S_{i}(t)$ are the semigroups defined in Section 2.3 generated by the unbounded operators $A_{i}$ and $w_{n}$ are Gaussian noises satisfying Assumption 4. $Z_{i}(t)$ also solves

$$
d Z_{i}(t)=B_{i} Z_{i}(t)+L_{i} Z_{i}(t)+\sum_{n=1}^{r} R_{n}(t) d w_{n}(t),
$$

where $B_{i}$ and $L_{i}$ are defined in Proposition 2.1. The mild solution of $Z_{i}$ solves

$$
Z_{i}(t)=\int_{0}^{t} T_{i}(t-s) L_{i} Z_{i}(s) d s+\tilde{Z}_{i}(t)
$$

where

$$
\tilde{Z}_{i}(t):=\int_{0}^{t} T_{i}(t-s) \sum_{n=1}^{r} R_{n}(s) d w_{n}(s)
$$

where $T_{i}(t)$ is the semigroup generated by the realization of $\mathcal{B}_{i}$ in $L^{2}(\mathcal{O})$ where $\mathcal{A}$ satisfies Assumption 3 and $w$ satisfies Assumption 4. Similar results can be found in Section 4 of [11. For $i \in\{1, \ldots, r\}$, let $T_{i}(t)$ be the semigroup on $H=L^{2}(\mathcal{O})$ generated by $B_{i}$ (see Proposition 2.1). There exists a kernel

$$
K_{i}(t, \xi, \eta):=\sum_{k=1}^{\infty} e^{-\alpha_{i, k} t} e_{i, k}(\xi) e_{i, k}(\eta)
$$


such that for any $\varphi \in L^{2}(\mathcal{O})$ and $t>0$,

$$
\left[T_{i}(t) \varphi\right](\xi)=\int_{\mathcal{O}} K_{i}(t, \xi, \eta) \varphi(\eta) d \eta
$$

The $T_{i}(t)$ semigroups have many useful smoothing properties including for $\gamma \in(0,1), p>1$,

$$
\begin{aligned}
& \left|T_{i}(t) \varphi\right|_{W^{\gamma, p}(\mathcal{O})} \leq C t^{-\frac{\gamma}{2}}|\varphi|_{L^{p}(\mathcal{O})} \\
& \left|T_{i}(t) \varphi\right|_{C(\overline{\mathcal{O}})} \leq C t^{-\frac{1}{2}}|\varphi|_{C^{-1}(\overline{\mathcal{O}})}
\end{aligned}
$$

We use the stochastic factorization method of Da Prato and Zabczyk [19]. For $\alpha \in\left(0, \frac{1}{2}\right)$, let

$$
\tilde{Z}_{i, \alpha}(\tau):=\int_{0}^{\tau}(\tau-s)^{-\alpha} T_{i}(\tau-s) \sum_{n=1}^{r} R_{n}(s) d w_{n}(s) .
$$

Then because $\int_{s}^{t}(t-\tau)^{\alpha-1}(\tau-s)^{-\alpha} d \tau=\frac{\pi}{\sin (\pi \alpha)}$,

$$
\tilde{Z}_{i}(t)=\frac{\sin (\pi \alpha)}{\pi} \int_{0}^{t}(t-\tau)^{1-\alpha} \tilde{Z}_{i, \alpha}(\tau) d \tau
$$

Lemma B.1. Let $\sigma \in E$. Let $R_{n} \in \mathscr{L}\left(L^{2}(\mathcal{O})\right)$ be given by $\left[R_{n} h\right](\xi)=$ $\sigma(\xi) h(\xi)$. Let $\left\{f_{n, j}\right\}$ and $\beta>0$ be as in Assumption 4. Then for any $i \in\{1, \ldots, r\}, t>0$, and $\xi \in \mathcal{O}$,

$$
\sum_{j=1}^{\infty}\left(\left(T_{i}(t) \sum_{n=1}^{r} R_{n} f_{n, j}\right)(\xi)\right)^{2} \leq C t^{-\beta}|\sigma|_{E}^{2} .
$$

Proof. Using the Kernel representation of the semigroup (B.6),

$$
\begin{aligned}
& \sum_{j=1}^{\infty}\left(\left(T_{i}(t) \sum_{n=1}^{r} R_{n} f_{n, j}\right)(\xi)\right)^{2} \\
& \leq \sum_{j=1}^{\infty}\left(\int_{\mathcal{O}} \sum_{n=1}^{r} K_{i}(t, \xi, \eta) \sigma_{n}(\eta) f_{n, j}(\eta) d \eta\right)^{2} \\
& \leq C \sum_{j=1}^{\infty} \sum_{n=1}^{r}\left(\int_{\mathcal{O}} K_{i}(t, \xi, \eta) \sigma_{n}(\eta) f_{n, j}(\eta) d \eta\right)^{2} .
\end{aligned}
$$


Because, for fixed $n,\left\{f_{n, j}\right\}_{n=1}^{\infty}$ is a complete orthonormal basis of $L^{2}(\mathcal{O})$,

$$
\begin{aligned}
& \sum_{n=1}^{r} \sum_{j=1}^{\infty}\left(\int_{\mathcal{O}} K_{i}(t, \xi, \eta) \sigma_{n}(\eta) f_{n, j}(\eta) d \eta\right)^{2} \\
& \leq \sum_{n=1}^{r} \int_{\mathcal{O}}\left(K_{i}(t, \xi, \eta) \sigma_{n}(\eta)\right)^{2} d \eta \\
& \leq C|\sigma|_{E}^{2} \int_{\mathcal{O}}\left(K_{i}(t, \xi, \eta)\right)^{2} d \eta .
\end{aligned}
$$

Because $\left\{e_{i, k}\right\}_{k=1}^{\infty}$ are an orthonormal basis of eigenfunctions, by (B.5)

$$
\begin{aligned}
& \int_{\mathcal{O}}\left(K_{i}(t, \xi, \eta)\right)^{2} d \eta \\
& \leq \int_{\mathcal{O}}\left(\sum_{k=1}^{\infty} e^{-\alpha_{i, k} t} e_{i, k}(\eta) e_{i, k}(\xi)\right)^{2} d \eta \\
& \leq \sum_{k=1}^{\infty} e^{-2 \alpha_{i, k} t}\left|e_{i, k}(\xi)\right|^{2}
\end{aligned}
$$

Let $c_{\beta}:=\sup _{x>0} x^{\beta} e^{-x}<+\infty$. Then.

$$
\begin{aligned}
& \sum_{k=1}^{\infty} e^{-2 \alpha_{i, k} t}\left|e_{i, k}(\xi)\right|^{2} \\
& \leq \sum_{k=1}^{\infty} 2^{-\beta} \alpha_{i, k}^{-\beta}\left|e_{i, k}\right|_{L^{\infty}(\mathcal{O})}^{2} t^{-\beta}\left(2 \alpha_{i, k} t\right)^{\beta} e^{-2 \alpha_{i, k} t} \\
& \leq c_{\beta} t^{-\beta} \sum_{k=1}^{\infty} \alpha_{i, k}^{-\beta}\left|e_{i, k}\right|_{L^{\infty}(\mathcal{O})}^{2} \\
& \leq C t^{-\beta} .
\end{aligned}
$$

The sum is finite by Assumption 4. The result now follows from (B.12).

Lemma B.2. For each $n \in\{1, \ldots, r\}$, let $\left\{f_{n, j}\right\}_{j=1}^{\infty}$ be the complete orthonormal basis of $L^{2}(\mathcal{O})$ and let $\left\{\lambda_{n, j}\right\}_{j=1}^{\infty}$ be the eigenvalues from Assumption 田 Let $\beta$ and $\rho$ be the constants from Assumption \&. There exists $C>0$ such that for any $\sigma \in E, R_{n}$ defined as in Lemma B.1, $i \in\{1, \ldots, r\}, t>0$, and $\xi \in \mathcal{O}$,

$$
\sum_{j=1}^{\infty}\left(\left(T_{i}(t) \sum_{n=1}^{r} R_{n} \lambda_{n, j} f_{n, j}\right)(\xi)\right)^{2} \leq C t^{-\frac{\beta(\rho-2)}{\rho}}|\sigma|_{E}^{2} .
$$


Proof. By the Hölder inequality with exponents $\frac{\rho}{2}$ and $\frac{\rho}{\rho-2}$,

$$
\begin{aligned}
& \sum_{j=1}^{\infty}\left(\left(T_{i}(t) \sum_{n=1}^{r} R_{n} \lambda_{n, j} f_{n, j}\right)(\xi)\right)^{2} \\
& \leq C \sum_{j=1}^{\infty} \sum_{n=1}^{r}\left(\left(T_{i}(t) R_{n} \lambda_{n, j} f_{n, j}\right)(\xi)\right)^{2} \\
& \leq C\left(\sum_{j=1}^{\infty} \sum_{n=1}^{r} \lambda_{n, j}^{\rho}\left|\left(T_{i}(t) R_{n} f_{n, j}\right)(\xi)\right|^{2}\right)^{\frac{2}{\rho}}\left(\sum_{j=1}^{\infty} \sum_{n=1}^{r}\left|\left(T_{i}(t) R_{n} f_{n, j}\right)(\xi)\right|^{2}\right)^{\frac{\rho-2}{\rho}}
\end{aligned}
$$

Because $T_{i}(t)$ is a contraction semigroup on $\tilde{E}$,

$$
\left|\left(T_{i}(t) R_{n} f_{n, j}\right)(\xi)\right| \leq \sup _{\eta \in \mathcal{O}}\left|\sigma_{n}(\eta) f_{n, j}(\eta)\right| \leq|\sigma|_{E}\left|f_{n, j}\right|_{L^{\infty}(\mathcal{O})} .
$$

Therefore,

$$
\sum_{j=1}^{\infty} \sum_{n=1}^{r} \lambda_{n, j}^{\rho}\left|\left(T_{i}(t) \sigma_{n} f_{n, j}\right)(\xi)\right|^{2} \leq\left(\sum_{j=1}^{\infty} \sum_{n=1}^{r} \lambda_{n, j}^{\rho}\left|f_{n, j}\right|_{L^{\infty}(\mathcal{O})}^{2}\right)|\sigma|_{E}^{2}
$$

The summation in the above display is finite by Assumption 4. By also applying (B.11),

$$
\begin{aligned}
& \left(\sum_{j=1}^{\infty} \sum_{n=1}^{r} \lambda_{n, j}^{\rho}\left|\left(T_{i}(t) \sigma_{n} f_{n, j}\right)(\xi)\right|^{2}\right)^{\frac{2}{\rho}}\left(\sum_{j=1}^{\infty}\left|\left(T_{i}(t) \sigma_{n} f_{n, j}\right)(\xi)\right|^{2}\right)^{\frac{\rho-2}{\rho}} \\
& \leq C t^{-\frac{\beta(\rho-2)}{\rho}}|\sigma|_{E}^{2} .
\end{aligned}
$$

Lemma B.3 (Estimates on $\left.\tilde{Z}_{i, \alpha}\right)$. Let $\sigma \in E_{T}$ and let $\tilde{Z}_{i, \alpha}$ be given by (B.9). For any $p>1$ there exists a constant $C>0$ such that for any $i \in\{1, \ldots, r\}$, $t>0, \xi \in D$,

$$
\mathbb{E}\left|\tilde{Z}_{i, \alpha}(t, \xi)\right|^{p} \leq C \mathbb{E}\left(\int_{0}^{t}(t-s)^{-2 \alpha-\frac{\beta(\rho-2)}{\rho}}|\sigma(s)|_{E}^{2} d s\right)^{\frac{p}{2}}
$$


Proof. By (2.19) and the BDG inequality, for any $\xi \in \mathcal{O}, t>0$,

$$
\begin{aligned}
& \mathbb{E}\left|\tilde{Z}_{i, \alpha}(t, \xi)\right|^{p} \\
& \leq C \mathbb{E}\left(\sum_{j=1}^{\infty} \int_{0}^{t}(t-s)^{-2 \alpha}\left|\left(T_{i}(t-s) \sum_{n=1}^{r} R_{n}(s) \lambda_{n, j} f_{n, j}\right)(\xi)\right|^{2} d s\right)^{\frac{p}{2}} .
\end{aligned}
$$

By (B.13),

$$
\mathbb{E}\left|\tilde{Z}_{i, \alpha}(t, \xi)\right|^{p} \leq C \mathbb{E}\left(\int_{0}^{t}(t-s)^{-2 \alpha-\frac{\beta(\rho-2)}{\rho}}|\sigma(s)|_{E}^{2} d s\right)^{\frac{p}{2}} .
$$

Theorem B.4 (Bounds on $\left.\tilde{Z}_{i}\right)$. Let $\tilde{Z}_{i}$ be given by (‥4). For $\alpha \in\left(0, \frac{1}{2}\left(1-\frac{\beta(\rho-2)}{\rho}\right)\right)$, $\gamma \in(0, \alpha), p>\max \left\{\frac{1}{\alpha-\gamma}, \frac{d}{\gamma}\right\}$, and $T>0$, there exists $C=C(\alpha, \gamma, p, T)>0$ such that

$$
\mathbb{E} \sup _{t \in[0, T]} \sup _{\xi \in \mathcal{O}}\left|\tilde{Z}_{i}(t, \xi)\right|^{p} \leq C \mathbb{E} \int_{0}^{T}\left(\int_{0}^{t}(t-s)^{-2 \alpha-\frac{\beta(\rho-2)}{\rho}}|\sigma(s)|_{E}^{2} d s\right)^{\frac{p}{2}} d t .
$$

Proof. The fractional Sobolev space $W^{\gamma, p}(\mathcal{O})$ embeds continuously into $\tilde{E}$ whenever $\gamma \in(0,1)$ and $\gamma p>d[21$. Therefore, by factorization (B.10]), the fractional Sobolev embedding, and the regularization of the $T_{i}$ semigroups (B.7),

$$
\begin{aligned}
& \mathbb{E} \sup _{t \in[0, T]} \sup _{\xi \in \mathcal{O}}\left|\tilde{Z}_{i}(t, \xi)\right|^{p} \\
& \leq C \mathbb{E} \sup _{t \in[0, T]}\left|\tilde{Z}_{i}(t)\right|_{W^{\gamma, p}(\mathcal{O})}^{p} \\
& \leq C \mathbb{E} \sup _{t \in[0, T]}\left|\int_{0}^{t}(t-s)^{\alpha-1} T_{i}(t-s) \tilde{Z}_{i, \alpha}(s) d s\right|_{W^{\gamma, p}(\mathcal{O})}^{p} \\
& \leq C \mathbb{E} \sup _{t \in[0, T]}\left(\int_{0}^{t}(t-s)^{\alpha-1-\gamma}\left|\tilde{Z}_{i, \alpha}(s)\right|_{L^{p}(\mathcal{O})} d s\right)^{p} .
\end{aligned}
$$

By the Hölder inequality,

$$
\leq C\left(\int_{0}^{T} s^{\frac{(\alpha-1-\gamma) p}{p-1}} d s\right)^{p-1} \mathbb{E} \int_{0}^{T}\left|\tilde{Z}_{i, \alpha}(t)\right|_{L^{p}(\mathcal{O})}^{p} d t
$$


By the fact that $p>\frac{1}{\alpha-\gamma}$, the first integral on the right-hand side is finite. By Lemma B.3. (B.16) follows.

Theorem B.5. Let $Z_{i}$ be given by (‥2). For $\alpha \in\left(0, \frac{1}{2}\left(1-\frac{\beta(\rho-2)}{\rho}\right)\right)$, $\gamma \in(0, \alpha), p>\max \left\{\frac{1}{\alpha-\gamma}, \frac{d}{\gamma}\right\}$, and $T>0$, there exists $C=C(\alpha, \gamma, p, T)>0$ such that

$$
\mathbb{E} \sup _{t \in[0, T]} \sup _{\xi \in \mathcal{O}}\left|Z_{i}(t, \xi)\right|^{p} \leq C \mathbb{E} \int_{0}^{T}\left(\int_{0}^{t}(t-s)^{-2 \alpha-\frac{\beta(\rho-2)}{\rho}}|\sigma(s)|_{E}^{2} d s\right)^{\frac{p}{2}} d t
$$

Proof. By (B.3) and (B.8),

$$
\begin{aligned}
& \left|Z_{i}(t)\right|_{\tilde{E}}^{p} \leq\left(\int_{0}^{t}\left|T_{i}(t-s) L_{i} Z_{i}(s)\right|_{\tilde{E}} d s\right)^{p}+C\left|\tilde{Z}_{i}(t)\right|_{\tilde{E}}^{p} \\
& \leq C\left(\int_{0}^{t}(t-s)^{-\frac{1}{2}}\left|Z_{i}(s)\right|_{\tilde{E}} d s\right)^{p}+C\left|\tilde{Z}_{i}(t)\right|_{\tilde{E}}^{p} .
\end{aligned}
$$

By the Hölder inequality for $p>2$,

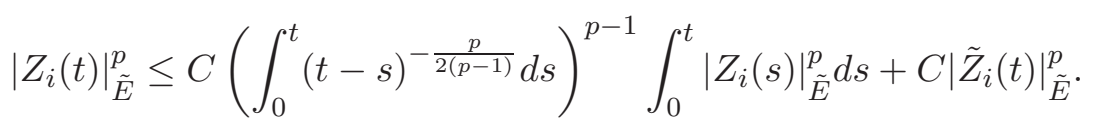

Taking expectation and applying Grönwall's inequality,

$$
\mathbb{E} \sup _{t \in[0, T]}\left|Z_{i}(t)\right|_{\tilde{E}}^{p} \leq C_{T, p} \sup _{t \in[0, T]}\left|\tilde{Z}_{i}(t)\right|_{\tilde{E}}
$$

The result follows from (B.16).

We finish this section with the analysis of similar terms where the stochastic noise has been replaced by a $L^{2}([0, T] \times \mathcal{O} \times\{1, \ldots, r\})$ control.

Theorem B.6. Let $u=\left(u_{1}, \ldots, u_{r}\right) \in L^{2}([0, T] \times \mathcal{O} \times\{1, \ldots, r\})$. Let $\sigma \in E_{T}$ and let $R$ be given by (B.1). Let $Y_{i}^{u}$ (weakly) solve

$$
d Y_{i}^{u}(t)=\left[A_{i} Y_{i}^{u}(t)+\sum_{n=1}^{r} R_{n}(t) Q_{n} u_{n}(t)\right] d t .
$$

Then $Y_{i}^{u} \in C([0, T] \times \mathcal{O})$ and there exists $C>0$, independent of $u$ and $\sigma$ such that

$$
\begin{aligned}
& \sup _{t \in[0, T]} \sup _{\xi \in \mathcal{O}}\left|Y_{i}^{u}(t, \xi)\right| \\
& \leq C N^{\frac{1}{2}} \sup _{t \in[0, T]}\left(\int_{0}^{t}(t-s)^{-\frac{\beta(\rho-2)}{\rho}}|\sigma(s)|_{E}^{2} d s\right)^{\frac{1}{2}} .
\end{aligned}
$$


where

$$
N=|u|_{L^{2}([0, T] \times \mathcal{O} \times\{1, \ldots, r\})}^{2}=\sum_{n=1}^{r} \int_{0}^{T} \int_{\mathcal{O}}\left|u_{n}(s, \xi)\right|^{2} d \xi d s
$$

Proof. As we did for the stochastic term, let

$$
\tilde{Y}_{i}^{u}(t)=\int_{0}^{t} T_{i}(t-s) \sum_{n=1}^{r} R_{n}(s) Q_{n} u_{n}(s) d s .
$$

This is the solution to

$$
d \tilde{Y}_{i}^{u}(t)=\left[B_{i} \tilde{Y}_{i}^{u}(t)+\sum_{n=1}^{r} R_{n}(t) Q_{n} u_{n}(t)\right] d t .
$$

We can rewrite (B.19) as

$$
\tilde{Y}_{i}^{u}(t)=\sum_{n=1}^{r} \sum_{j=1}^{\infty} \int_{0}^{t} T_{i}(t-s) R_{n}(s) \lambda_{n, j} f_{n, j}\left\langle u_{n}(s), f_{n, j}\right\rangle_{L^{2}(\mathcal{O})} d s .
$$

By the Hölder inequality, for any $\xi \in \mathcal{O}$,

$$
\begin{aligned}
\left|\tilde{Y}_{i}^{u}(t, \xi)\right| \leq\left(\sum_{n=1}^{r}\right. & \left.\sum_{j=1}^{\infty} \int_{0}^{t}\left|\left(T_{i}(t-s) R_{n}(s) \lambda_{n, j} f_{n, j}\right)(\xi)\right|^{2} d s\right)^{\frac{1}{2}} \\
& \times\left(\sum_{n=1}^{r} \sum_{j=1}^{\infty} \int_{0}^{t}\left\langle u_{n}(s), f_{n, j}\right\rangle_{L^{2}(\mathcal{O})}^{2} d s\right)^{\frac{1}{2}}
\end{aligned}
$$

By (B.13) and the fact that $\left\{f_{n, j}\right\}_{j=1}^{\infty}$ is a complete orthonormal basis of $L^{2}(\mathcal{O})$, for any $t>0$ and $\xi \in \mathcal{O}$,

$$
\left|\tilde{Y}_{i}^{u}(t, \xi)\right| \leq C\left(\int_{0}^{t}(t-s)^{-\frac{\beta(\rho-2)}{\rho}}|\sigma(s)|_{E}^{2} d s\right)^{\frac{1}{2}}\left(\sum_{n=1}^{r} \int_{0}^{t} \int_{\mathcal{O}}\left|u_{n}(s, \eta)\right|^{2} d \eta d s\right)^{\frac{1}{2}} .
$$

The continuity of $\tilde{Y}_{i}^{u}$ in space in time can be shown by standard arguments (see, for example, [38]).

Then $Y_{i}^{u}(t)$ solves

$$
Y_{u}^{i}(t)=\int_{0}^{t} T_{i}(t-s) L_{i} Y_{i}^{u}(s) d s+\tilde{Y}_{i}^{u}(t) .
$$

By (B.8),

$$
\left|Y_{i}^{u}(t)\right|_{\tilde{E}} \leq \int_{0}^{t}(t-s)^{-\frac{1}{2}}\left|Y_{i}^{u}(s)\right|_{\tilde{E}} d s+\left|\tilde{Y}_{i}^{u}(t)\right|_{\tilde{E}} .
$$

The result follows by Grönwall's inequality. 


\section{Bounds on the fixed-point mapping that are uniform with respect to the initial condition.}

Let $E$ and $E_{T}$ be defined as in Section 2, For any $z \in E_{T}$ with $z(0)=0$ and $x \in E$ let $\mathcal{M}_{x}(z)$ be the solution to the fixed-point problem

$$
\mathcal{M}_{x}(z)(t):=\mathcal{M}(S(\cdot) x+z)
$$

where $\mathcal{M}$ is the fixed point mapping defined in (6.1) and $S$ is the semigroup defined in Section 2.3. The following result establishes bounds on $\mathcal{M}_{x}$ that are independent of $x$ when the vector field $f$ features super-linear dissipativity (see Assumption 6).

Theorem C.1. Assume Assumptions 1, 3, and 6. For any $t>0$ there exists $C_{t}>0$ ( $C_{t}$ increases as $t$ increases), independent of $x \in E$, such that for any $z \in E_{t}$ with $z(0)=0$ and any $x \in E$,

$$
\left|\mathcal{M}_{x}(z)(t)\right|_{E} \leq C_{t}\left(1+t^{-\frac{1}{m-1}}+|z|_{E_{t}}\right) .
$$

Proof. Let

$$
v(t)=\mathcal{M}_{x}(z)(t)-z(t) .
$$

$v(t)$ solves the integral equation

$$
v(t)=S(t) x+\int_{0}^{t} S(t-s) F(v(s)+z(s)) d s .
$$

Therefore, $v$ is weakly differentiable and solves

$$
\frac{\partial}{\partial t} v_{i}(t, \xi)=\mathcal{A}_{i} v_{i}(t, \xi)+g_{i}\left(v_{i}(t, \xi)+z_{i}(t, \xi)\right)+h_{i}(v(t, \xi)+z(t, \xi)) .
$$

Using the arguments of Theorem 7.7 in [19] and Proposition 6.2.2 of [10] we may assume without loss of generality that $v$ is strongly differentiable.

By Proposition A.1, and Assumption [6, for any $i_{t} \in\{1, \ldots, r\}$ and $\xi_{t} \in \mathcal{O}$ such that

$$
\begin{aligned}
& \qquad|v(t)|_{E}=\left|v_{i_{t}}\left(t, \xi_{t}\right)\right| \\
& \frac{d^{-}}{d t}|v(t)|_{E} \\
& \leq \mathcal{A}_{i_{t}} v_{i_{t}}\left(t, x_{t}\right) \operatorname{sign}\left(v_{i_{t}}\left(t, \xi_{t}\right)\right) \\
& \quad+g_{i_{t}}\left(t, \xi_{t}, v_{i_{t}}\left(t, \xi_{t}\right)+z_{i_{t}}\left(t, \xi_{t}\right)\right) \operatorname{sign}\left(v_{i_{t}}\left(t, \xi_{t}\right)\right) \\
& \quad+h_{i_{t}}\left(t, \xi, v\left(t, \xi_{t}\right)+z\left(t, \xi_{t}\right)\right) \operatorname{sign}\left(v_{i_{t}}\left(t, \xi_{t}\right)\right) .
\end{aligned}
$$


Because $\left(i_{t}, \xi_{t}\right)$ is a maximizer and $\mathcal{A}_{i_{t}}$ is an elliptic operator, the concavity of a function at is maximum/minimum implies that

$$
\mathcal{A}_{i_{t}} v_{i_{t}}\left(t, x_{t}\right) \operatorname{sign}\left(v_{i_{t}}\left(t, \xi_{t}\right)\right) \leq 0 .
$$

We will estimate this derivative when $|v(t)|_{E}$ is large. If $|v(t)|_{E} \geq 2 c_{0}+$ $2|z|_{E_{t}}$, where $c_{0}$ is from Assumption [6, then

$$
\left|v_{i_{t}}\left(t, \xi_{t}\right)+z_{i_{t}}\left(t, \xi_{t}\right)\right| \geq\left|v_{i_{t}}\left(t, \xi_{t}\right)\right|-\left|z_{i_{t}}\left(t, \xi_{t}\right)\right| \geq \frac{1}{2}\left|v_{i_{t}}\left(t, \xi_{t}\right)\right|>c_{0}
$$

and

$$
\operatorname{sign}\left(v_{i_{t}}\left(t, \xi_{t}\right)+z_{i_{t}}\left(t, \xi_{t}\right)\right)=\operatorname{sign}\left(v_{i_{t}}\left(t, \xi_{t}\right)\right) .
$$

Therefore, (4.8), (C.7), and (C.8) guarantee that

$g_{i_{t}}\left(t, \xi_{t}, v_{i_{t}}\left(t, \xi_{t}\right)+z_{i_{t}}\left(t, \xi_{t}\right)\right) \operatorname{sign}\left(v_{i_{t}}\left(t, \xi_{t}\right)\right) \leq-\frac{\mu}{2^{m}}\left|v_{i_{t}}\left(t, \xi_{t}\right)\right|^{m}=-\frac{\mu}{2^{m}}|v(t)|_{E}^{m}$.

By (2.11) in Assumption 1, for any $t \in[0, T]$,

$$
\begin{aligned}
h_{i_{t}}\left(t, \xi, v\left(t, \xi_{t}\right)+z\left(t, \xi_{t}\right)\right) \operatorname{sign}\left(v_{i_{t}}\left(t, \xi_{t}\right)\right) & \leq L(t)\left(1+|v(t)+z(t)|_{E}\right) \\
& \leq L(t)\left(1+\frac{3}{2}|v(t)|_{E}\right) .
\end{aligned}
$$

There exists a constant $C_{T}>c_{0}$, depending on $\mu, c_{0}$, and $L(T)$ such that whenever $|v(t)|_{E}>C_{T}+2|z|_{E_{T}}$,

$$
-\frac{\mu}{2^{m}}|v(t)|_{E}^{m}+L(T)\left(1+\frac{3}{2}|v(t)|_{E}\right) \leq-\frac{\mu}{2^{m+1}}|v(t)|_{E}^{m} .
$$

Therefore, whenever $t \in[0, T]$ and

$$
|v(t)|_{E} \geq C_{T}+2|z|_{E_{T}}
$$

it follows from the above estimates that

$$
\frac{d^{-}}{d t}|v(t)|_{E} \leq-\frac{\mu}{2^{m+1}}|v(t)|_{E}^{m}
$$

We now separate this analysis into two cases: $|v(0)|_{E} \leq C_{T}+2|z|_{E_{T}}$ and $|v(0)|_{E}>C_{T}+2|z|_{E_{T}}$.

If $|v(0)|_{E} \leq C_{T}+2|z|_{E_{T}}$, then $|v(t)|_{E} \leq C_{T}+2|z|_{E_{T}}$ for all $t \in[0, T]$ because $\frac{d^{-}}{d t}|v(t)|_{E}<0$ when $|v(t)|_{E}=C_{T}+2|z|_{E_{T}}$. The negative leftderivative implies that $|v(t)|_{E}$ cannot ever reach the value $C_{T}+2|z|_{E_{T}}$ if it starts below that value. 
On the other hand, when $|v(0)|_{E}>C_{T}+2|z|_{E_{T}}$, let $\tau=\inf \{t \in[0, T]$ : $\left.|v(t)|_{E} \leq C_{T}+2|z|_{E_{T}}\right\}$. Observe that (C.13) holds for all $t \in[0, \tau]$. By a comparison principle, there exists $C>0$ such that for all $t \in[0, \tau]$

$$
|v(t)|_{E} \leq C t^{-\frac{1}{m-1}} \text { for all } t \in[0, \tau]
$$

uniformly with respect to initial data. Then for $t \in(\tau, T]$,

$$
|v(t)|_{E} \leq C_{T}+2|z|_{E_{T}}
$$

because $|v(t)|_{E}$ cannot exceed this value once it goes below it.

These calculations show that, independent of initial data, $|v(t)|_{E}$ is bounded by

$$
|v(t)|_{E} \leq \max \left\{C t^{-\frac{1}{m-1}}, C_{T}+2|z|_{E_{T}}\right\} \text { for all } t \in[0, T] .
$$

Finally,

$$
\left|M_{x}(z)(t)\right|_{E} \leq|v(t)|_{E}+|z(t)|_{E} \leq C_{t}\left(1+t^{-\frac{1}{m-1}}+|z|_{E_{t}}\right)
$$

\section{References}

[1] Hakima Bessaih and Annie Millet, Large deviation principle and inviscid shell models, Electronic Journal of Probability 14 (2009), 2551-2579.

[2] Large deviations and the zero viscosity limit for $2 D$ stochastic Navier-Stokes equations with free boundary, SIAM Journal on Mathematical Analysis 44 (2012), no. 3, 1861-1893.

[3] Anup Biswas and Amarjit Budhiraja, Exit time and invariant measure asymptotics for small noise constrained diffusions, Stochastic Processes and their Applications 121 (2011), no. 5, 899-924.

[4] Zdzisław Brzeźniak, Sandra Cerrai, and Mark Freidlin, Quasipotential and exit time for $2 D$ stochastic Navier-Stokes equations driven by space time white noise, Probability Theory and Related Fields 162 (2015), no. 3-4, 739-793.

[5] Amarjit Budhiraja and Paul Dupuis, A variational representation for positive functionals of infinite dimensional Brownian motion, Probability and Mathematical Statistics 20 (2000), no. 1, 39-61.

[6] Amarjit Budhiraja, Paul Dupuis, and Markus Fischer, Large deviation properties of weakly interacting processes via weak convergence methods, The Annals of Probability 40 (2012), no. 1, 74-102.

[7] Amarjit Budhiraja, Paul Dupuis, and Vasileios Maroulas, Large deviations for infinite dimensional stochastic dynamical systems, The Annals of Probability 36 (2008), no. 4, 1390-1420. 
[8] L Large deviations for stochastic flows of diffeomorphisms, Bernoulli 16 (2010), no. $1,234-257$.

[9] Caroline Cardon-Weber, Large deviations for a Burgers'-type SPDE, Stochastic Processes and their Applications 84 (1999), no. 1, 53-70.

[10] Sandra Cerrai, Second order PDE's in finite and infinite dimension: a probabilistic approach, Vol. 1762, Springer Science \& Business Media, 2001.

[11] Stochastic reaction-diffusion systems with multiplicative noise and nonLipschitz reaction term, Probability Theory and Related Fields 125 (2003), no. 2, 271-304.

[12] _ A Khasminskii type averaging principle for stochastic reaction-diffusion equations, The Annals of Applied Probability 19 (2009), no. 3, 899-948.

[13] Sandra Cerrai and Michael Röckner, Large deviations for stochastic reaction-diffusion systems with multiplicative noise and non-Lipshitz reaction term, The Annals of Probability 32 (2004), no. 1B, 1100-1139.

[14] Sandra Cerrai and Michael Salins, Smoluchowski-Kramers approximation and large deviations for infinite dimensional gradient systems, Asymptotic Analysis 88 (2014), no. 4, 201-215.

[15] _ Smoluchowski-Kramers approximation and large deviations for infinitedimensional nongradient systems wih applications to the exit problem, The Annals of Probaility 44 (2016), no. 4, 2591-2642.

[16] Fabien Chenal and Annie Millet, Uniform large deviations for parabolic SPDEs and applications, Stochastic Processes and their Applications 72 (1997), no. 2, 161-186.

[17] Pao-Liu Chow and José-Luis Menaldi, Exponential estimates in exit probability for some diffusion processes in Hilbert spaces, Stochastics: An International Journal of Probability and Stochastic Processes 29 (1990), no. 3, 377-393.

[18] Igor Chueshov and Annie Millet, Stochastic 2D hydrodynamical type systems: well posedness and large deviations, Applied Mathematics \& Optimization 61 (2010), no. 3, 379-420.

[19] Giuseppe Da Prato and Jerzy Zabczyk, Stochastic equations in infinite dimensions, Cambridge University Press, 2014.

[20] Amir Dembo and Ofer Zeitouni, Large deviations techniques and applications, Vol. 38, Springer Science \& Business Media, 2009.

[21] Eleonora Di Nezzaa, Giampiero Palatuccia, and Enrico Valdinocia, Hitchhiker's guide to the fractional sobolev spaces, Bull. Sci. Math 136 (2012), no. 5, 521-573.

[22] Jinqiao Duan and Annie Millet, Large deviations for the Boussinesq equations under random influences, Stochastic processes and their Applications 119 (2009), no. 6, 2052-2081.

[23] Lawrence C Evans, Graduate studies in mathematics, Partial differential equations, 1998.

[24] William G Faris and Giovanni Jona-Lasinio, Large fluctuations for a nonlinear heat equation with noise, Journal of Physics A: Mathematical and General 15 (1982), no. 10, 3025-3055.

[25] Jin Feng and Thomas G Kurtz, Large deviations for stochastic processes, American Mathematical Soc., 2006. 
[26] Mohammud Foondun and Leila Setayeshgar, Large deviations for a class of semilinear stochastic partial differential equations, Statistics \& Probability Letters 121 (2017), $143-151$.

[27] Mark Freidlin, Random perturbations of reaction-diffusion equations: the quasideterministic approximation, Transactions of the American Mathematical Society 305 (1988), no. 2, 665-697.

[28] Mark Freidlin and Alexander Wentzell, Random perturbations of dynamical systems, Vol. 260, Springer Science \& Business Media, 2012.

[29] Eric Gautier, Uniform large deviations for the nonlinear Schrödinger equation with multiplicative noise, Stochastic Processes and their Applications 115 (2005), no. 12, 1904-1927.

[30] Martin Hairer and Hendrik Weber, Large deviations for white-noise driven, nonlinear stochastic PDEs in two and three dimensions, Annales de la Faculté des sciences de Toulouse: Mathématiques, 2015, pp. 55-92.

[31] Gopinath Kallianpur and Jie Xiong, Large deviations for a class of stochastic partial differential equations, The Annals of Probability 24 (1996), no. 1, 320-345.

[32] Wei Liu, Large deviations for stochastic evolution equations with small multiplicative noise, Applied Mathematics and Optimization 61 (2010), no. 1, 27-56.

[33] Wei Liu, Michael Röckner, and Xiang-Chan Zhu, Large deviation principles for the stochastic quasi-geostrophic equations, Stochastic Processes and their Applications 123 (2013), no. 8, 3299-3327.

[34] Yan Lv and AJ Roberts, Large deviation principle for singularly perturbed stochastic damped wave equations, Stochastic Analysis and Applications 32 (2014), no. 1, 50-60.

[35] Carlo Marinelli and Michael Röckner, On uniqueness of mild solutions for dissipative stochastic evolution equations, Infinite Dimensional Analysis, Quantum Probability and Related Topics 13 (2010), no. 03, 363-376.

[36] Chi Mo and Jiaowan Luo, Large deviations for stochastic differential delay equations, Nonlinear Analysis: Theory, Methods \& Applications 80 (2013), 202-210.

[37] Víctor Ortiz-López and Marta Sanz-Solé, A Laplace principle for a stochastic wave equation in spatial dimension three, Stochastic analysis 2010, 2011, pp. 31-49.

[38] Amnon Pazy, Semigroups of linear operators and applications to partial differential equations, Vol. 44, Springer Science \& Business Media, 2012.

[39] Szymon Peszat, Large deviation principle for stochastic evolution equations, Probability Theory and Related Fields 98 (1994), no. 1, 113-136.

[40] Jiagang Ren, Siyan Xu, and Xicheng Zhang, Large deviations for multivalued stochastic differential equations, Journal of Theoretical Probability 23 (2010), no. 4, 11421156.

[41] Jiagang Ren and Xicheng Zhang, Freidlin-Wentzell's large deviations for stochastic evolution equations, Journal of Functional Analysis 254 (2008), no. 12, 3148-3172.

[42] Michael Röckner, Tusheng Zhang, and Xicheng Zhang, Large deviations for stochastic tamed 3D Navier-Stokes equations, Applied Mathematics \& Optimization 61 (2010), no. 2, 267-285.

[43] Michael Salins, Equivalences and counterexamples between several definitions of the uniform large deviations principle, Probability Surveys 16 (2019), 99-142. 
[44] Michael Salins, Amarjit Budhiraja, and Paul Dupuis, Uniform large deviation principles for banach space valued stochastic evolution equations, Transactions of the American Mathematical Society 372 (2019), no. 12, 8363-8421.

[45] Richard Sowers, Large deviations for a reaction-diffusion equation with non-Gaussian perturbations, The Annals of Probability 20 (1992), no. 1, 504-537.

[46] SS Sritharan and P Sundar, Large deviations for the two-dimensional Navier-Stokes equations with multiplicative noise, Stochastic Processes and their Applications 116 (2006), no. 11, 1636-1659.

[47] Chengfeng Sun, Hongjun Gao, Jinqiao Duan, and Björn Schmalfuß, Rare events in the Boussinesq system with fluctuating dynamical boundary conditions, Journal of Differential Equations 248 (2010), no. 6, 1269-1296.

[48] SR Srinivasa Varadhan, Large deviations and applications, Vol. 46, SIAM.

[49] Tiange $\mathrm{Xu}$ and Tusheng Zhang, White noise driven SPDEs with reflection: existence, uniqueness and large deviation principles, Stochastic Processes and Their Applications 119 (2009), no. 10, 3453-3470.

[50] Jianliang Zhai and Tusheng Zhang, Large deviations for stochastic models of twodimensional second grade fluids, Applied Mathematics \& Optimization 75 (2017), no. $3,471-498$. 